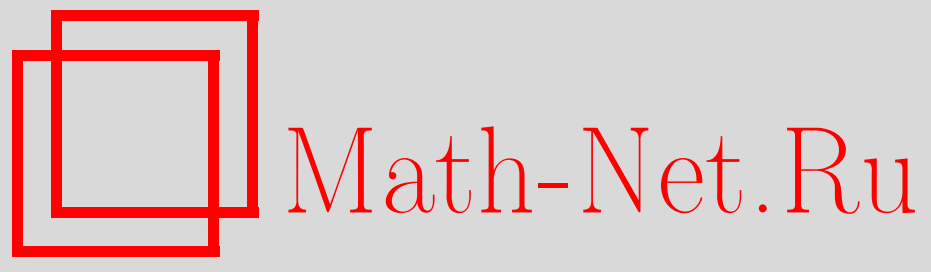

В. А. Малышев, С. А. Пирогов, Обратимость и необратимость в стохастической химической кинетике, $У М H$, 2008, том 63, выпуск 1, 3-36

DOI: https://doi.org/10.4213/rm8517

Использование Общероссийского математического портала Math-Net.Ru подразумевает, что вы прочитали и согласны с пользовательским соглашением http://www .mathnet.ru/rus/agreement

Параметры загрузки:

IP : 3.91 .87 .62

26 апреля 2023 г., 10:12:38

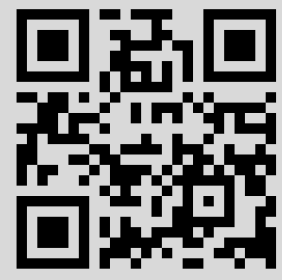


УДК 519.2

\title{
Обратимость и необратимость в стохастической химической кинетике
}

\author{
В. А. Малышев, С. А. Пирогов
}

Рассматриваются математические модели, связанные со стохастической химической кинетикой: модели типа среднего поля классической химической кинетики, а также локальные модели. Основной упор делается на различные понятия типа обратимости, их следствия (уравнения типа Больцмана, флуктуации, соотношения Онзагера и др.) и связи с возникновением необратимости.

Библиография: 37 названий.

\section{СОДЕРЖАНИЕ}

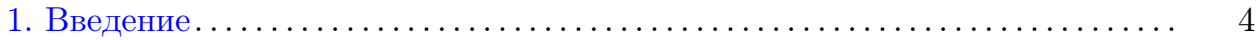

2. Стохастическая химическая кинетика...................... 5

2.1. Стохастическое и классическое описание химических реакций .. 5

2.2. Унитарность и инвариантность пуассоновской меры ........ 7

2.3. Обратимость в теории вероятностей ................... 8

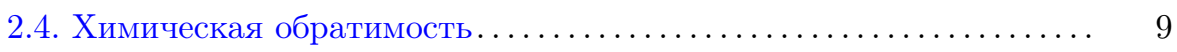

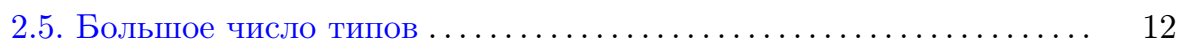

3. Сложность поведения и необратимость ...................... 13

3.1. Неподвижные точки . . . . . . . . . . . . . . . . . . . 13

3.2. Флуктуации и соотношения Онзагера.................... 15

3.3. Как получаются необратимые реакции .................. 19

4. Стохастические локальные модели . . . . . . . . . . . . . . . . 22

4.1. Стохастические модели со смешанной динамикой ............ 22

4.2. Уравнение Больцмана с химическими реакциями ............ 24

4.3. Простейшие модели с транспортом на решетке............. 29

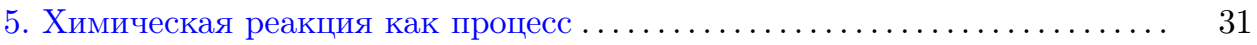

5.1. Обратимость во времени в физике..................... 31

5.2. Классическое гамильтоново рассеяние ................ 32

5.3. Скорости реакций из локальной теории ................ 33

5.4. Динамика с немгновенными реакциями................. 33

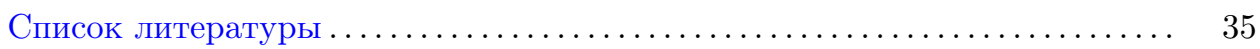

(C) В. А. МАлышев, С. А. Пирогов, 2008 


\section{1. Введение}

Известно, что замкнутая физическая система ведет себя достаточно просто, а именно стремится к равновесию. Одна из причин этого - обратимость динамики во времени. В биологии мы имеем совсем другое, более сложное, поведение. Обычный метод биологических и химических теорий в объяснении сложных биологических явлений состоит в подборе необратимых систем химических реакций. Довольно ясно, что так можно объяснить любое поведение, что снижает ценность теории. В то же время необратимая система реакций формально может быть выведена из (обратимых) законов физики только при определенных предельных переходах.

В данном обзоре мы обсуждаем математические задачи, связанные с обратимостью и необратимостью систем химических реакций.

В разделе 2 мы разбираем связи между различными вероятностными характеристиками системы химических реакций. Мы даем классическое и стохастическое описание химических реакций. Классическая химическая кинетика составляет ядро любых теоретических и прикладных исследований в биологии. Стохастическая кинетика всегда носила более теоретический характер, но в последнее время подчеркивается ее прикладной аспект, см. [1]-[3]. Мы изучаем связь инвариантности пуассоновской меры с тремя важнейшими вариантами обратимости для систем химических реакций: химической обратимостью, обратимостью в смысле теории марковских процессов и унитарностью. В заключение мы касаемся случая большого числа типов, еще мало развитого.

Основной вопрос - как ведут себя системы химических реакций при больших временах. В разделе 3 показывается, что, в предположении обратимости, это поведение довольно просто - система сходится к неподвижной точке, т. е. состоянию равновесия. Поведение в окрестности неподвижной точки описывается обычно двумя физическими теориями: линейные возмущения, которые ведут к соотношениям симметрии Онзагера, и еще более малые возмущения - стохастические флуктуации. Мы излагаем строгие версии этих теорий. Наконец, мы рассматриваем два основных пути возникновения необратимости в системах химических реакций - транспорт и временной скейлинг.

В физике необратимость обычно возникает при предельном переходе к уравнению Больцмана. Стохастическая и классическая химическая кинетика являются типичными примерами теорий, которые в физике называются теориями среднего поля. В разделе 4 мы рассмотрим разные возможности введения локальности в системах бесконечного числа частиц. Мы выделим три различных типа скейлингов, которые дают в пределе детерминированные квазилинейные уравнения в частных производных с нелинейным членом, аналогичным правой части уравнений (3) классической химической кинетики:

1) канонический скейлинг с $M \rightarrow \infty$ в химической кинетике, где вместе с этим частицы перемещаются в пространстве;

2) предел Больцмана-Грэда в системе частиц с локальными химическими реакциями;

3) стандартные гидродинамические скейлинги для систем частиц на решетке, совершающих случайные блуждания. 
В ньютоновой и квантовой механике понятие обратимости другое, и возникает естественный вопрос о связи между этими понятиями. Мы касаемся этого в разделе 5. С другой стороны, во всех моделях химической кинетики, рассматриваемых в разделах $2-4$, реакция считается мгновенным событием. На самом же деле реакция занимает определенный промежуток времени, который как в классических, так и в квантовых моделях является формально бесконечным. Это означает, что теория рассеяния и химическая кинетика имеют дело с разными временными шкалами, достаточно разделенными между собой. Тем не менее, интенсивности реакций в химический кинетике должны получаться из математической теории рассеяния. В конце раздела 5 мы вводим понятие метастабильной частицы, т. е. частицы с конечным временем жизни, не как особенность (резонанс) в спектре конечночастичного оператора, а как некое локальное образование в бесконечночастичной системе.

\section{2. Стохастическая химическая кинетика}

2.1. Стохастическое и классическое описание химических реакций. Пусть имеется конечное число типов молекул $A_{v}, v=1, \ldots, V$. Химическая реакция $r$ определяется векторами $d_{-}(r)=\left\{d_{-}(v, r)\right\}, d_{+}(r)=\left\{d_{+}(v, r)\right\}$ стехиометрических коэффициентов, где неотрицательные целые числа $d_{-}(v, r)-$ кратности субстратов $A_{v}$ типа $v, d_{+}(v, r)$ - кратности продуктов типа $v$, и записывается формально как

$$
\sum_{v} d_{-}(v, r) A_{v} \rightarrow \sum_{v} d_{+}(v, r) A_{v}
$$

Рассмотрим систему химических реакций, т. е. марковский процесс $\mathscr{M}$ с состояниями $n=\left(n_{1}, \ldots, n_{V}\right)$ с $R$ переходами (типами реакций) $r=1, \ldots, R$ :

$$
n_{v} \rightarrow n_{v}+d_{+}(v, r)-d_{-}(v, r), \quad v=1, \ldots, V,
$$

где $n_{v}$ - число молекул типа $v$.

Здесь мы должны сделать следующее замечание. Ниже в разделе 5, особенно в п. 5.3, мы обсуждаем, как данная стохастическая модель химической кинетики связана с основными физическими принципами. Заметим, однако, что строгого вывода в достаточно общих предположениях не существует. Здесь возникают две проблемы. Первая связана с тем, что в стохастической химической кинетике рассматривается шкала времени, более грубая в сравнении с временем одиночной реакции. Время одиночной реакции могло бы быть вычислено с помощью квантовой теории рассеяния. Однако эти вычисления даже для простейших реакций достаточно сложны, и, более того, разделение этих двух шкал нигде не было проделано в строгом виде. Для вывода локальных моделей, рассматриваемых ниже в разделе 4, из первых принципов этого было бы достаточно. Однако в разделах 2 и 3 мы рассматриваем модель среднего поля. И вторая проблема в том, как связана модель среднего поля с локальными моделями. Мы не даем ответа на этот вопрос, который возникает всегда, когда в физике переходят к моделям типа среднего поля. Скажем, однако, что получающиеся в локальном случае и в случае среднего поля предельные 
уравнения типа Больцмана показывают, что отличие состоит в перенормировке скорости реакции. Промежуточным случаем могла бы быть модель Каца, в которой молекуле разрешается реагировать не с любой другой молекулой, а только с находящимися от нее на расстоянии не большем $\varepsilon L$, где $L$ - диаметр системы.

Реакция $r$, т. е. переход (1), имеет интенсивность (в химической кинетике называемую скоростью реакции [4])

$$
\lambda_{r}(n)=\lambda_{r}\left(n \rightarrow n-d_{-}(r)+d_{+}(r)\right)=M^{-m_{-}(r)+1} a_{r} \prod_{v \in I(r)} n_{v} \cdots\left(n_{v}-d_{-}(v, r)+1\right),
$$

где $a_{r}$ - фиксированные числа (константы скорости - в химической кинетике), $M-$ масштабный коэффициент и

$$
m_{-}(r)=\sum_{v} d_{-}(v, r), \quad I(r)=\left\{v: d_{-}(v, r)>0\right\} .
$$

Показатель степени $M$ в (2) соответствует так называемому каноническому скейлингу, см. [5].

Заметим, что уравнение (2) выражает известный в химической кинетике закон действующих масс.

Мы будем также рассматривать выделенные реакции "вход" и "выход"

$$
\varnothing \rightarrow A_{v}, \quad A_{v} \rightarrow \varnothing
$$

с интенсивностями $M a_{v \text {, in }}$ и $a_{v, \text { out }} n_{v}$ соответственно. Если в системе нет таких реакций, будем называть ее замкнутой. В противном случае будем называть ее открытой.

Отметим, что история стохастической химической кинетики начинается с работы [6], последующее ее математическое развитие состояло в основном в исследовании систем с малыми $R$ и $V$ (ссылки см. в [7], [8]).

Сходимость к уравнениям классической кинетики. Классическая кинетика получается из стохастической как предел при $M \rightarrow \infty$. Точнее говоря, мы рассматриваем семейство процессов $n_{v}(t)=n_{v}^{(M)}(t)$, зависящих от параметра $M$. Мы приводим здесь хорошо известный результат (см. ссылки в [5]) в несколько более общем виде, включая возможный вход и выход.

Теорема 1. Предположим, что в началъный момент времени предел

$$
c_{v}(0)=\lim _{M \rightarrow \infty} \frac{n_{v}(0)}{M}
$$

существует для всех $v$. Тогда для всех $v$ u $t>0$ предель по вероятности

$$
c_{v}(t)=\lim _{M \rightarrow \infty} \frac{n_{v}(t)}{M}
$$

существуют и удовлетворяют уравнениям

$$
\frac{d c_{v}(t)}{d t}=\sum_{r: v \in O(r)} d_{+}(v, r) a_{r} \prod_{w \in I(r)} c_{w}^{d_{-}(w, r)}-\sum_{r: v \in I(r)} d_{-}(v, r) a_{r} \prod_{w \in I(r)} c_{w}^{d_{-}(w, r)},
$$


где

$$
O(r)=\left\{v: d_{+}(v, r)>0\right\}
$$

(т.е. стандартным уравнениям химической кинетики).

Законы сохранения обеспечивают компактность. Пусть $w_{b v}$ - число атомов типа $b$ в молекуле типа $v$. Тогда для всех реакций $r$ (кроме входа и выхода) и всех типов атомов $b$ имеет место равенство

$$
\sum_{v \in I(r)} d_{-}(v, r) w_{b v}=\sum_{v \in O(r)} d_{+}(v, r) w_{b v} .
$$

Заметим, что предельные члены входа и выхода имеют вид

$$
\sum_{v}\left(a_{v, \text { in }}-a_{v, \text { out }} c_{v}\right)
$$

Если нет законов сохранения, то в общем случае траектория может уходить на бесконечность или приходить в неподвижную точку за конечное время. Доказательство теоремы для этого случая см. в [5].

2.2. Унитарность и инвариантность пуассоновской меры. Условие инвариантности пуассоновской меры для марковского процесса $\mathscr{M}$, задаваемой формулой

$$
\mu(n)=\prod_{v} \frac{\bar{b}_{v}^{n_{v}}}{n_{v} !} e^{-\bar{b}_{v}}, \quad \bar{b}_{v}=M b_{v},
$$

где $b_{v}$ - некоторые фиксированные параметры, запишется в виде равенства выходных,

$$
F_{\text {out }}=\mu(n) \sum_{r} \lambda_{r}(n)
$$

и входных,

$$
F_{\text {in }}=\sum_{r} \mu\left(n^{\prime}\right) \lambda_{r}\left(n^{\prime}\right), \quad n^{\prime}=n+d_{-}(r)-d_{+}(r),
$$

"потоков вероятности" для любого заданного состояния $n=\left(n_{1}, \ldots, n_{V}\right)$. Заметим, что ввиду (2) суммирование в правой части (4) автоматически делается только по допустимым реакциям, т. е. по таким, где $n_{v}-d_{-}(v, r) \geqslant 0$, а в правой части (5) - по $r$ таким, что $n_{v}^{\prime}-d_{-}(v, r)=n_{v}-d_{+}(v, r) \geqslant 0$.

Учитывая равенство

$$
\frac{\mu\left(n^{\prime}\right)}{\mu(n)}=\prod_{v} \bar{b}_{v}^{n_{v}^{\prime}-n_{v}} \frac{n_{v} !}{n_{v}^{\prime} !},
$$

из условия $F_{\text {in }}=F_{\text {out }}$, деля на $\mu(n)$ и пользуясь однозначностью определения $n_{v}^{\prime}$ по $n_{v}$ и $r$, т. е. равенством $n_{v}^{\prime}-d_{-}(v, r)=n_{v}-d_{+}(v, r)$, получим

$$
\begin{array}{r}
\sum_{r} M^{-m_{-}(r)+1} a_{r} \prod_{v} \bar{b}_{v}^{n_{v}^{\prime}-n_{v}} \frac{n_{v} !}{n_{v}^{\prime} !} n_{v}^{\prime} \cdots\left(n_{v}^{\prime}-d_{-}(v, r)+1\right) \\
=\sum_{r} M^{-m_{-}(r)+1} a_{r} \prod_{v \in I(r)} n_{v} \cdots\left(n_{v}-d_{-}(v, r)+1\right),
\end{array}
$$


или

$$
\begin{gathered}
\sum_{r} M^{-m_{-}(r)+1} a_{r} \prod_{v \in I(r)} \bar{b}_{v}^{d_{-}(v, r)-d_{+}(v, r)} n_{v} \cdots\left(n_{v}-d_{+}(v, r)+1\right) \\
=\sum_{r} M^{-m_{-}(r)+1} a_{r} \prod_{v \in I(r)} n_{v} \cdots\left(n_{v}-d_{-}(v, r)+1\right) .
\end{gathered}
$$

И наконец,

$$
\begin{gathered}
\sum_{r} M^{-m_{+}(r)+1} a_{r} \prod_{v \in I(r)} b_{v}^{d_{-}(v, r)-d_{+}(v, r)} n_{v} \cdots\left(n_{v}-d_{+}(v, r)+1\right) \\
=\sum_{r} M^{-m_{-}(r)+1} a_{r} \prod_{v \in I(r)} n_{v} \cdots\left(n_{v}-d_{-}(v, r)+1\right)
\end{gathered}
$$

где $m_{+}(r)=m_{-}(r)-\sum_{v \in I(r)}\left(d_{-}(v, r)-d_{+}(v, r)\right)$.

ТЕорема 2. Следующие утверждения эквивалентны:

1) пуассоновская мера $\mu$ инвариантна относителъно заданной системы химических реакиий;

2) (условие Штюкельберга, или условие унитарности) для любого вектора $d$ имеет место равенство

$$
\sum_{r: d_{-}(r)=d} a_{r} \prod_{v \in I(r)} b_{v}^{d_{-}(v, r)}=\sum_{r: d_{+}(r)=d} a_{r} \prod_{v \in I(r)} b_{v}^{d_{-}(v, r)} .
$$

ДокАЗАТЕЛЬство. Ввиду обратимости предыдущей выкладки мы доказали, что условие 1) эквивалентно условию (6). Воспользуемся теперь тем, что для равенства нулю многочлена от $n_{v}$ необходимо и достаточно, чтобы коэффициенты при его квазимономах были равны нулю. Два равных монома определяются вектором

$$
d=d_{-}(r)=d_{+}(r)
$$

Кроме того, можно сократить масштабные множители, заметив, что при этом $m_{-}(r)=m_{+}(r)$.

Заметим, что условие (7) имеет смысл и для классической детерминированной химической кинетики, где его можно формулировать так: сумма скоростей реакций, рождающих группу частиц, характеризуемую вектором $d$ (и только эту группу), равна сумме скоростей реакций, которые уничтожают эту (и только эту) группу.

Отметим еще такой факт, указанный А.Н. Рыбко: при условии наличия пуассоновской инвариантной меры обращение времени в процессе стохастической химической кинетики приводит к процессу того же типа, но, вообще говоря, с другим множеством реакций.

2.3. Обратимость в теории вероятностей. В теории вероятностей есть свое понятие обратимости во времени [9], [10]. Стационарный случайный процесс $X_{t}$ называется обратимым (во времени), если конечномерные распределения процессов $X_{t}$ и $Y_{t}=X_{-t}$ совпадают. Для марковских процессов (например 
со счетным пространством состояний и непрерывным временем) это определение эквивалентно следующему условию детального баланса:

$$
\pi_{i} \lambda_{i j}=\pi_{j} \lambda_{j i}
$$

где $\lambda_{i j}$ - интенсивности переходов, $\pi$ - инвариантная мера марковского процесса. Последнее определение может быть применено также к однородным по времени, но нестационарным, т. е. к нулевым возвратным и невозвратным, марковским процессам, если существует (уже бесконечная) неотрицательная мера $\pi$ на пространстве состояний, удовлетворяющая условию (8). Это мы будем также называть обратимостью относительно меры $\pi$. При этом если $\pi$ равна единице в каждой точке, то обратимость эквивалентна симметричности матрицы $\left(\lambda_{i j}\right)$. Поэтому любая обратимая цепь получается из цепи с симметрической матрицей переходов преобразованием

$$
\lambda_{i j} \rightarrow w_{i} \lambda_{i j} w_{j}^{-1}, \quad w_{i}=\sqrt{\pi_{i}} .
$$

Критерий Колмогорова обратимости марковского процесса относительно меры $\pi$ состоит в выполнении равенств

$$
\lambda_{i_{1} i_{2}} \lambda_{i_{2} i_{3}} \cdots \lambda_{i_{n} i_{1}}=\lambda_{i_{1} i_{n}} \lambda_{i_{n} i_{n-1}} \cdots \lambda_{i_{2} i_{1}}
$$

для любой последовательности состояний $i_{1}, \ldots, i_{n}$. При выполнении этого условия, пользуясь определением (8), меру $\pi$ легко построить как

$$
\pi_{i_{n}}=\pi_{0} a_{0 i_{1}} a_{i_{1} i_{2}} \cdots a_{i_{n-1} i_{n}}, \quad a_{i j}=\frac{\lambda_{i j}}{\lambda_{j i}},
$$

для любой последовательности состояний $i_{1}, \ldots, i_{n}$, где

$$
\pi_{0}^{-1}=1+\sum_{k} a_{G(0, k)},
$$

где сумма берется по всем состояниям $k \neq 0, G(0, k)$ - некоторый путь из 0 в $k$, т. е. последовательность состояний $i_{0}, i_{1}, \ldots, i_{n}$ такая, что $i_{0}=0, i_{n}=k$, и

$$
a_{G(0, k)}=a_{0 i_{1}} a_{i_{1} i_{2}} \cdots a_{i_{n-1} i_{n}} .
$$

2.4. Химическая обратимость. Понятие химической обратимости можно ввести в общем контексте марковских цепей. Предположим, что на пространстве состояний $X$ задано некоторое конечное множество А семейств переходных интенсивностей $\lambda_{m n}^{\alpha}, m, n \in X, \alpha \in \mathbf{A}$, марковских цепей с непрерывным временем. Элементы множества А будем называть "химическими реакциями". Предположим, что на А определена операция инволюции - обратная реакция $\alpha \rightarrow \alpha^{\prime} \neq \alpha$, причем $\left(\alpha^{\prime}\right)^{\prime}=\alpha$.

Определим цепь Маркова $\xi_{\mathbf{A}}$ на $X$ интенсивностями переходов

$$
\lambda_{m n}=\sum_{\alpha} \lambda_{m n}^{\alpha} .
$$

Назовем эту цепь химически обратимой, если существует распределение вероятностей $\pi_{n}$ на $X$ такое, что для всех $\alpha$ выполнено условие

$$
\pi_{n} \lambda_{n m}^{\alpha}=\pi_{m} \lambda_{m n}^{\alpha^{\prime}}
$$


В классической химической кинетике обратная реакция $r^{\prime}$ к реакции $r$ однозначно определяется условиями $d_{-}(r)=d_{+}\left(r^{\prime}\right), d_{-}\left(r^{\prime}\right)=d_{+}(r)$.

Для классической кинетики условие детального баланса - совпадение скоростей прямой и обратной реакций - имеет вид

$$
a_{r} \prod_{v \in I(r)} b_{v}^{d_{-}(v, r)}=a_{r^{\prime}} \prod_{v \in I\left(r^{\prime}\right)} b_{v}^{d_{-}\left(v, r^{\prime}\right)} .
$$

Мы увидим сейчас различие между условиями (8) и (9).

ТЕОрема 3. Пусть дана система химических реакций, где для каждой реакиии r существует обратная $r^{\prime}$. Тогда следующие условия эквивалентны:

1) соответствующий марковский прочесс химически обратим;

2) система химических реакиий обратима (как случайный прочесс) относительно некоторой пуассоновской меры.

ДокАЗАТЕльство. 1) $\Rightarrow 2$ ). Для пары $r, r^{\prime}$ двух взаимно обратных реакций условие химической обратимости имеет вид

$$
\pi_{n} a_{r} \prod_{v} \frac{n_{v} !}{\left(n_{v}-d_{-}(v, r)\right) !}=\pi_{n^{\prime}} a_{r^{\prime}} \prod_{v} \frac{n_{v}^{\prime} !}{\left(n_{v}^{\prime}-d_{+}(v, r)\right) !}
$$

для некоторой меры $\left\{\pi_{n}\right\}$ и для любых $n$ и $n^{\prime}$ таких, что $n^{\prime}=n-d_{-}(r)+d_{+}(r)$. Если обозначить

$$
z_{n}=\log \left(\pi_{n} \prod_{v} n_{v} !\right)
$$

то вместо (10) будем иметь

$$
z_{n}-z_{n^{\prime}}=\log \frac{a_{r^{\prime}}}{a_{r}}
$$

Обозначим $l_{r}$ правую часть (11), которая в химии называется константой равновесия. Если в рассматриваемой системе химических реакций $\mathbf{A}$ все векторы

$$
d=d(r)=d_{-}(r)-d_{+}(r), \quad r \in \mathbf{A},
$$

различны, то можно считать $l_{r}$ функцией $l(d)$ от $d=d(r)$. Однако может случиться так, что разные реакции имеют одинаковые векторы $d(r)$. Тем не менее, функция $l(d)$ имеет следующие свойства:

(а) из вида правой части (11) и из того, что $d(r)=-d\left(r^{\prime}\right)$, следует $l(d)=$ $-l(-d)$

(b) из вида левой части (11) следует, что если для некоторой последовательности реакций $r_{1}, \ldots, r_{k}$ имеет место равенство $\sum_{i=1}^{k} d\left(r_{i}\right)=0$, то

$$
\sum_{i=1}^{k} l\left(d\left(r_{i}\right)\right)=0
$$

Из этих двух свойств следует, что $l(d)$ может быть продолжена до (аддитивного) гомоморфизма $\phi$ аддитивной подгруппы $Q \subset \mathbb{Z}^{V}$ в $\mathbb{R}$, где $V$ - число типов молекул в системе, а $Q$ порождается всеми векторами $d(r)$, т. е.

$$
\phi(0)=0, \quad \phi(x+y)=\phi(x)+\phi(y) .
$$


Этот гомоморфизм может быть продолжен до аддитивного гомоморфизма $\mathbb{Z}^{V}$ в $\mathbb{R}$. Любой такой гомоморфизм имеет вид

$$
\phi(x)=\sum_{v} \alpha_{v} x_{v}
$$

для некоторых вещественных чисел $\alpha_{v}$.

Теперь можно проверить, что пуассоновская мера

$$
p_{n}=\prod_{v} \frac{\alpha_{v}^{n_{v}}}{n_{v} !} e^{-\alpha_{v}}
$$

удовлетворяет условию (10). Имеем

$$
w_{n}=\log \left(p_{n} \prod_{v} n_{v} !\right)=-\sum_{v} \alpha_{v} n_{v}+\text { const },
$$

и, следовательно, для любой пары конфигураций $n, n^{\prime}$ таких, что $n^{\prime}=n-$ $d_{-}(r)+d_{+}(r)$, получим

$$
w_{n}-w_{n^{\prime}}=\sum_{v} \alpha_{v} d_{v}(r)=l(d(r)), \quad l(d(r))=\log \frac{a_{r^{\prime}}}{a_{r}} .
$$

Отсюда следует, что мера $p_{n}$ удовлетворяет уравнению (11) или, что эквивалентно, уравнению (10). И, значит, рассматриваемая цепь Маркова имеет эту меру в качестве стационарного распределения и является обратимой по этой мере.

$2) \Rightarrow 1)$. Условие обратимости марковского процесса относительно пуассоновской меры (12) состоит в том, что для любых векторов $n, n^{\prime}$

$$
p_{n} \sum_{r} a_{r} \prod_{v} \frac{n_{v} !}{\left(n_{v}-d_{-}(r)\right) !}=p_{n^{\prime}} \sum_{r^{\prime}} a_{r^{\prime}} \frac{n_{v}^{\prime} !}{\left(n_{v}^{\prime}-d_{+}(r)\right) !},
$$

где суммирование производится по всем реакциям $r$ таким, что $n_{v}-n_{v}^{\prime}=d_{-}(r)-$ $d_{+}(r)$ и $n_{v} \geqslant d_{-}(r)$ для всех $v$. Из (13) следует

$$
\prod_{v} \alpha_{v}^{n_{v}} \sum_{r} a_{r} \prod_{v} \frac{1}{\left(n_{v}-d_{-}(r)\right) !}=\prod_{v} \alpha_{v}^{n_{v}^{\prime}} \sum_{r^{\prime}} a_{r^{\prime}} \prod_{v} \frac{1}{\left(n_{v}^{\prime}-d_{+}(r)\right) !} .
$$

Отсюда

$$
\sum_{r} a_{r} \prod_{v} \alpha_{v}^{d_{-}(r)-d_{+}(r)} \frac{1}{\left(n_{v}-d_{-}(r)\right) !}=\sum_{r^{\prime}} a_{r^{\prime}} \prod_{v} \frac{1}{\left(n_{v}-d_{-}(r)\right) !}
$$

при прежних соглашениях о суммировании. Иначе говоря,

$$
\begin{gathered}
\sum_{r} a_{r} \prod_{v} \alpha_{v}^{d_{-}(r)-d_{+}(r)} n_{v} \cdots\left(n_{v}-d_{-}(r)+1\right) \\
=\sum_{r} a_{r} \prod_{v} n_{v} \cdots\left(n_{v}-d_{-}(r)+1\right)
\end{gathered}
$$


для фиксированных $n_{v}$ и $d(r)$. Поскольку $n_{v}$ произвольны, то последнее уравнение может выполняться, только если для вектора $d_{-}(r)$ (и соответствующего вектора $\left.d_{+}(r)=d(r)+d_{-}(r)\right)$ выполняется равенство

$$
a_{r} \prod_{v} \alpha_{v}^{d_{-}(r)-d_{+}(r)}=a_{r^{\prime}} .
$$

Это условие, очевидно, эквивалентно химической обратимости для пуассоновской меры (10) и условию детального баланса (9). Теорема 3 доказана.

ЗАмЕчАНИЕ 4. Пример Шлёгля (см. п. 3.1) показывает, что из обычной обратимости не следует инвариантность пуассоновской меры.

2.5. Большое число типов. Здесь мы не даем четких формулировок, но указываем на интересные классы задач. Модели, где $V$ велико, физически могут соответствовать двум совсем разным ситуациям, которые мы рассмотрим ниже. В первой ситуации система разделена на большое число клеток. Можно представлять, что клетки образуют разбиение пространства. В каждой клетке количество типов веществ (молекул) ограничено. При этом любое вещество реагирует только с веществами из своей или соседних клеток, число соседних клеток также равномерно ограничено. Во втором случае, наоборот, число типов веществ в каждой клетке может быть как угодно велико. При этом если количество типов атомов ограничено, то количество атомов в веществе может быть очень большим. Более того, наиболее интересные ситуации возникают, когда интенсивности реакций велики, иначе говоря, у этих веществ должно быть короткое время жизни. Тогда все вещества активно влияют на эволюцию. Вещества с коротким временем жизни соответствуют метастабильным молекулам, или, как сейчас принято их называть в химии, кластерам.

Локально конечные сети реакций. Мы введем их для случая, когда

$$
\sum_{v} d_{+}(v, r), \quad \sum_{v} d_{-}(v, r)
$$

равномерно ограничены по $r$, а

$$
\sum_{r} d_{+}(v, r), \quad \sum_{r} d_{-}(v, r)
$$

ограничены равномерно по $v$. Тогда можно взять $V$ счетным.

Бесконечная система уравнений классической кинетики выводится так же, как и выше. Конечно, $M$ опять имеет смысл объема, но не общего, а локального объема каждой клетки.

Условие унитарности и теорема 2 переносятся на бесконечный случай. Условие марковской обратимости хорошо известно для процессов с локальным взаимодействием, наша система является таковым. В этом же духе может быть обобщено условие химической обратимости.

Модель с кластерами большого объема. Пусть есть только один тип элементарных атомов и кластеры различаются только размером. Точнее говоря, мы рассматриваем разбиения множества из $N$ элементов-атомов на подмножества, которые мы называем кластерами. Пусть $m_{v}-$ число кластеров 
размера $v=1, \ldots, V$, т. е. из $v$ атомов, при этом есть закон сохранения

$$
\sum_{v} v m_{v}=N
$$

но само $N$ может быть случайным, число типов $V$ фиксируется, но потом мы интересуемся асимптотикой $V \rightarrow \infty$. Определим цепь Маркова, состояниями которой являются векторы $m=\left(m_{1}, \ldots, m_{N}\right)$. Возможные реакции:

1) $r_{n}$ - присоединение частицы к кластеру размера $n>1$ :

$$
m_{1} \rightarrow m_{1}-1, \quad m_{n} \rightarrow m_{n}-1, \quad m_{n+1} \rightarrow m_{n+1}+1,
$$

и соединение $r_{1}$ двух частиц:

$$
m_{1} \rightarrow m_{1}-2, \quad m_{2} \rightarrow m_{2}+1
$$

2) $r_{n}^{\prime}$ - отсоединение частицы от кластера размера $n>2$ :

$$
m_{1} \rightarrow m_{1}+1, \quad m_{n} \rightarrow m_{n}-1, \quad m_{n-1} \rightarrow m_{n-1}+1,
$$

и распад $r_{1}^{\prime}$ кластера из двух частиц:

$$
m_{1} \rightarrow m_{1}+2, \quad m_{2} \rightarrow m_{2}-1 .
$$

Их интенсивности $a_{r_{n}}=a_{n}, a_{r_{n}^{\prime}}=a_{n}^{\prime}$ не зависят, конечно, от $V$ и $N$. Предположим, что выполнено условие химической обратимости для любой пары $r_{n}, r_{n+1}^{\prime}$. Обозначим через $\pi$ стационарное пуассоновское распределение этой цепи с параметрами $b_{v}$. Тогда условие химической обратимости дает для всех $n=1, \ldots, V$

$$
a_{n} b_{1} b_{n}=a_{n+1}^{\prime} b_{n+1} .
$$

Эти соотношения позволяют строить множество примеров. В частности, для любого заданного набора положительных чисел $b_{1}, b_{2}, \ldots$ однозначно определяются отношения скоростей прямых и обратных реакций $a_{n} / a_{n}^{\prime}$. Ряд таких примеров с конкретными $a_{n}, a_{n}^{\prime}$ рассмотрен в книге $\Phi$. Келли [11], где речь идет о малом ансамбле.

\section{3. Сложность поведения и необратимость}

3.1. Неподвижные точки. Рассмотрим снова уравнения (3) классической химической кинетики. Оказывается, что в предположении унитарности (а значит, и в предположении обратимости) их решения имеют достаточно простое поведение.

Энтропия Больцмана положительного вектора концентраций $c=\left(c_{1}, \ldots, c_{V}\right)$ относительно некоторой фиксированной неотрицательной меры $c^{0}$ на $\{1, \ldots, V\}$ определяется как

$$
H(c)=H\left(c, c^{0}\right)=\sum_{v} c_{v} \log \frac{c_{v}^{0}}{c_{v}}+\sum_{v} c_{v}=\sum_{v} c_{v} \log \frac{e c_{v}^{0}}{c_{v}} .
$$

Заметим, что множитель $е$ не может быть отброшен, если число частиц не сохраняется. Интересно, что это выражение сходно с выражением для энтропии смеси идеальных газов (см. [12], [13]). 
ТЕОРема 5. Пусть для заданной системы химических реакиий существует вектор $c^{0}>0$, относительно которого система удовлетворяет условию унитарности (7). Тогда имеют место следующие утверждения:

1) для любого решения $c_{v}(t)$ кинетических уравнений (3) с любым начальным условием $c_{v}(0)$ имеет место возрастание энтропии, т.е.

$$
\frac{d H(c(t))}{d t} \geqslant 0
$$

2) при $t \rightarrow \infty$ любое решение $c(t)$ уравнений (3) стремится $\kappa$ некоторой неподвижной точке $c_{\infty}$, которая, вообще говоря, зависит от с(0);

3) условие унитарности выполняется для всех неподвижных точек уравнений (3);

4) если для некоторой неподвижной точки выполнено условие детального баланса, то оно выполнено и для любой другой неподвижной точки.

Доказательства этих утверждений даны в [5]; см. также [14], [15].

Системы с одним типом частиц. Без условия унитарности такое простое поведение возможно лишь в простейших случаях. Показательны следующие примеры с одним типом частиц. Для одного типа частиц с реакциями $r: d_{-}(r) \rightarrow d_{+}(r)$ имеем

$$
\frac{d c(t)}{d t}=\sum_{r}\left(d_{+}(r)-d_{-}(r)\right) a_{r} c^{d_{-}(r)}(t),
$$

т. е. в правой части может быть произвольный многочлен от $c$. Нас интересуют только неотрицательные решения этого уравнения. Рассмотрим частный случай, а именно модель Шлёгля [16], где есть только реакции типов $0 \rightarrow 1$, $1 \rightarrow 0,2 \rightarrow 3,3 \rightarrow 2$. Обозначим соответствующие $a_{r}$ через $a_{01}, a_{10}, a_{23}, a_{32}$. Нетрудно показать, что условие унитарности выполняется только в следующих трех случаях:

1) “вход-выход": $a_{23}=a_{32}=0$;

2) $a_{01}=a_{10}=0$ (будем называть этот случай замкнутой моделью Шлёгля);

3) если

$$
\frac{a_{23}}{a_{32}}=\frac{a_{01}}{a_{10}} .
$$

Можно показать также, что в этих случаях выполняется не только условие унитарности, но и условие детального баланса (9).

В общем случае, когда первые четыре коэффициента многочлена (14) отличны от нуля, на положительной полуоси может быть либо одна, либо три неподвижные точки. Из трех неподвижных точек правая и левая устойчивы, а средняя неустойчива.

ЗАмЕчАниЕ 6. В случае нескольких типов частиц класс многочленов, получаемых от реакций с законами сохранения и без них, достаточно широк. Для примера рассмотрим для этого случая множество $R(I)$ всех реакций $r$, где $I=I(r)=O(r)$. Для них многочлен произволен, а именно имеет вид

$$
\sum_{r \in R(I)} a_{r}\left(d_{+}(v, r)-d_{-}(v, r)\right) \prod_{v \in I} c_{v}^{d_{-}(v, r)} .
$$


Отсюда следует, что необратимые системы химических реакций могут иметь сколь угодно сложное поведение. Так, известно много примеров с циклами [17]. По-видимому, можно доказать, что системами химических реакций может быть моделировано любое алгоритмическое поведение (любой конечный автомат). С одной стороны, это свидетельствует о неограниченных возможностях биосистем, а с другой - может служить легким источником подгонки теории к эксперименту.

3.2. Флуктуации и соотношения Онзагера. Поведению систем в окрестности неподвижной точки (т. е. равновесного распределения) в статистической физике уделялось большое внимание. Так, в теории флуктуаций равновесной динамики (т.е. динамики, сохраняющей равновесную меру) речь идет об окрестности порядка $1 / \sqrt{M}$ концентраций $c_{v}$. Можно также делать линеаризацию динамической системы в малой окрестности - это один из способов вывести соотношения симметрии Онзагера. Дадим точные математические определения.

Линеаризация и соотношения Онзагера. Рассмотрим случай малых возмущений, т. е. случай, когда для начальных данных $n_{v}(0)$ предел

$$
c_{v}(0)=\lim _{M \rightarrow \infty} \frac{n_{v}^{(M)}(0)}{M}
$$

лежит в малой $\varepsilon$-окрестности неподвижной точки.

Линеаризация уравнений (3) около выбранного решения $c_{v}(t)$ дает следующие уравнения для вариаций $x_{v}(t)$ решения $c_{v}(t)$ :

$$
\frac{d x_{v}}{d t}=\sum_{r} a_{r}\left(d_{+}(v, r)-d_{-}(v, r)\right) \sum_{u} d_{-}(u, r) c_{u}^{d_{-}(u, r)-1} x_{u} \prod_{w \neq u} c_{w}^{d_{-}(w, r)} .
$$

В частности, если в качестве решения берется неподвижная точка $c_{v}(t)=\bar{c}_{v}$, то имеем систему линейных дифференциальных уравнений с постоянными коэффициентами:

$$
\frac{d x_{v}}{d t}=\sum_{r} a_{r}\left(d_{+}(v, r)-d_{-}(v, r)\right) \sum_{u} d_{-}(u, r) \bar{c}_{u}^{d_{-}(u, r)-1} x_{u} \prod_{w \neq u} \bar{c}_{w}^{d_{-}(w, r)} .
$$

Запишем ее в виде

$$
\frac{d x_{v}}{d t}=\sum_{u} \lambda_{v u} x_{u}
$$

где матрица коэффициентов имеет вид

$$
\lambda_{v u}=\sum_{r} a_{r}\left(d_{+}(v, r)-d_{-}(v, r)\right) d_{-}(u, r) \bar{c}_{u}^{d_{-}(u, r)-1} \prod_{w \neq u} \bar{c}_{w}^{d_{-}(w, r)} .
$$

ТЕОРема 7. При условии химической обратимости имеют место следующие соотношения Онзагера:

$$
\lambda_{v u} \bar{c}_{u}=\lambda_{u v} \bar{c}_{v}
$$


ДокАЗАТЕЛЬСтво. Из (17) имеем

$$
\lambda_{v u} \bar{c}_{u}=\sum_{r} a_{r}\left(d_{+}(v, r)-d_{-}(v, r)\right) d_{-}(u, r) \prod_{w} \bar{c}_{w}^{d_{-}(w, r)} .
$$

Предположим теперь, что выполнено условие детального баланса для прямой и обратной реакций $r, r^{\prime}$ :

$$
a_{r} \prod_{w} \bar{c}_{w}^{d_{-}(w, r)}=a_{r^{\prime}} \prod_{w} \bar{c}_{w}^{d_{+}(w, r)} .
$$

Тогда вклад в выражение (19) от двух взаимно обратных реакций $r, r^{\prime}$ равен

$$
\begin{aligned}
& a_{r}\left(d_{+}(v, r)-d_{-}(v, r)\right) d_{-}(u, r) \prod_{w} \bar{c}_{w}^{d_{-}}(w, r) \\
& \quad+a_{r^{\prime}}\left(d_{-}(v, r)-d_{+}(v, r)\right) d_{+}(u, r) \prod_{w} \bar{c}_{w}^{d_{+}(w, r)}
\end{aligned}
$$

Вклад от тех же двух реакций в выражение для $\lambda_{u v} \bar{c}_{v}$, аналогичное (19), равен

$$
\begin{aligned}
& a_{r}\left(d_{+}(u, r)-d_{-}(u, r)\right) d_{-}(v, r) \prod_{w} \bar{c}_{w}^{d_{-}(w, r)} \\
& \quad+a_{r^{\prime}}\left(d_{-}(u, r)-d_{+}(u, r)\right) d_{+}(v, r) \prod_{w} \bar{c}_{w}^{d_{+}(w, r)} .
\end{aligned}
$$

Легко видеть, что выражения (21) и (22) совпадают. Действительно, слагаемые, содержащие произведения $d_{-}(u, r) d_{-}(v, r)$ и $d_{+}(u, r) d_{+}(v, r)$, в $(21)$ и $(22)$ идентичны, а оставшиеся слагаемые равны соответственно

$$
a_{r} d_{+}(v, r) d_{-}(u, r) \prod_{w} \bar{c}_{w}^{d_{-}(w, r)}+a_{r^{\prime}} d_{-}(v, r) d_{+}(u, r) \prod_{w} \bar{c}_{w}^{d_{+}(w, r)}
$$

И

$$
a_{r} d_{+}(u, r) d_{-}(v, r) \prod_{w} \bar{c}_{w}^{d_{-}(w, r)}+a_{r^{\prime}} d_{-}(u, r) d_{+}(v, r) \prod_{w} \bar{c}_{w}^{d_{+}(w, r)} .
$$

Но ввиду условия детального баланса (20) эти выражения также совпадают. Теорема 7 доказана.

Энтропия. Если выполнено условие унитарности Штюкельберга, то можно довольно просто интуитивно объяснить возрастание энтропии $H(c)$ со временем. Действительно, в этом случае пуассоновская мера $Q$ с параметрами $M \bar{c}_{i}$ является инвариантной. Можно указать модель, где динамика сохраняет, точно (см. п. 4.1) или приближенно, класс всех пуассоновских мер. Таким образом, пуассоновская мера $P_{t}$ может меняться во времени, при этом ее параметры $M c_{i}(t)$ некоторым образом зависят от времени. Тогда информационное расстояние Кульбака-Лейблера имеет вид

$$
\rho_{\mathrm{KL}}\left(Q, P_{t}\right)=\int P_{t} \ln \frac{P_{t}}{Q}=-M \widetilde{H}(c),
$$

где

$$
\widetilde{H}(c)=H(c)-\sum \bar{c}_{i}
$$


(очевидно, что $\left.H(\bar{c})=\sum_{i} \bar{c}_{i}\right)$. С другой стороны, хорошо известно, что для любого марковского процесса, при некоторых технических ограничениях, $\rho_{\mathrm{KL}}\left(Q, P_{t}\right)$ монотонно (хотя, возможно, не строго монотонно) убывает со временем. В этом смысле теорема 5 является уточнением этого общего утверждения.

Энтропию $\widetilde{H}(c)$ можно также интерпретировать в терминах вероятностей больших уклонений (где $n$ - произвольная конфигурация):

$$
\lim _{M \rightarrow \infty} \frac{\ln Q(n)}{M}=\widetilde{H}(c),
$$

если $n / M \rightarrow c$.

Флуктуации. Флуктуации концентраций в момент $t$, при заданном $M$, определяются следующим образом:

$$
\xi_{v}^{(M)}(t)=\xi_{v}^{\left(M, n_{v}(0)\right)}(t)=\frac{n_{v}(t)-\left\langle n_{v}(t)\right\rangle}{\sqrt{M}},
$$

где $\langle\cdot\rangle$ - среднее. Этот процесс зависит, кроме того, от начальных данных $n_{v}(0)$ или от $\left\langle n_{v}(t)\right\rangle$. Равновесные флуктуации соответствуют процессу $\xi_{v}(t+s)$ в пределе $s \rightarrow \infty$, когда есть устойчивая неподвижная точка $c_{v}^{\text {fixed }}=\bar{c}_{v}$ и

$$
c_{v}^{\text {fixed }}=\lim _{M \rightarrow \infty} \frac{n_{v}(0)}{M} .
$$

Теорема 8. Пустъ марковский прочесс $\xi_{v}^{(M)}(t)$ для заданного М стационарен и выполнено условие (24). Тогда предел (в смысле конечномерных распределений) $\left(\xi_{v}(t)\right)$ векторного процесса $\left(\xi_{v}^{(M)}(t)\right)$ при $M \rightarrow \infty$ является процессом Орнштейна-Уленбека со средним 0 и матрищей ковариащий

$$
D_{v v^{\prime}}\left(t-t^{\prime}\right)=\left\langle\xi_{v}(t) \xi_{v^{\prime}}\left(t^{\prime}\right)\right\rangle
$$

определяемой формулой (26) ниже.

ДокАЗАТЕЛЬСтво. Для любого фиксированного момента времени из пуассоновости распределения вектора $\left(\xi_{v}^{(M)}(t)\right)$ следует, что он сходится по распределению к гауссовскому вектору $\left(\xi_{v}(t)\right)$ с независимыми компонентами, при этом

$$
\left\langle\xi_{v}(t)\right\rangle=0, \quad\left\langle\xi_{v}^{2}(t)\right\rangle=\bar{c}_{i},
$$

т. е. его плотность вектора $\left(\xi_{v}(t)\right)$ имеет вид

$$
\rho=\text { const } \cdot \exp \left(-\frac{1}{2} \sum_{i} \frac{x_{i}^{2}}{\bar{c}_{i}}\right) .
$$

При этом квадратичная форма в показателе экспоненты есть не что иное, как квадратичная часть разложения Тейлора функции $H(\bar{c})$ в точке $c=\bar{c}$. Иначе говоря, матрица $\beta^{-1}$ ковариаций является диагональной с диагональными элементами $\bar{c}_{i}$. По определению матрица $\gamma$ кинетических коэффициентов равна $\lambda \beta^{-1}$. Таким образом, она имеет вид

$$
\gamma_{i j}=\lambda_{i j} \bar{c}_{j}
$$

Согласно теореме 7, эта матрица, как и следовало ожидать, симметрична. 
Перейдем к описанию временных корреляций. Если предел процесса $\xi_{v}^{(M)}(t)$ существует, то он является стационарным и обратимым процессом, так как таковым является процесс $\xi_{v}^{(M)}(t)$ для всех $M$. Из центральной предельной теоремы следует больше (см. [18], [19]), а именно, что в пределе $M \rightarrow \infty$ этот процесс является гауссовским марковским с генератором

$$
L=\sum_{v, w} D_{v w} \frac{\partial^{2}}{\partial x_{v} \partial x_{w}}+\sum_{v} b_{v} \frac{\partial}{\partial x_{v}},
$$

где

$$
b_{v}(x)=\sum_{u} \lambda_{v u} x_{u},
$$

т. е. скорость сноса определяется линеаризованными кинетическими уравнениями (16), ср. [20].

Это можно проверить прямой выкладкой: снос в точке $\left(n_{1}, \ldots, n_{V}\right)$ равен

$$
\begin{aligned}
S_{v}\left(n_{1}, \ldots, n_{V}\right)= & \sum_{r}\left(d_{+}(v, r)-d_{-}(v, r)\right) a_{r} M^{-m(r)+1} \\
& \times \prod_{w} n_{w}\left(n_{w}-1\right) \cdots\left(n_{w}-d_{-}(w, r)+1\right) .
\end{aligned}
$$

Обозначим

$$
c_{v}^{(M)}=\frac{n_{v}}{M}, \quad s_{v}=\frac{S_{v}}{M} .
$$

Рассмотрим устойчивую неподвижную точку $\bar{c}_{v}^{(M)}=\bar{n}_{v} / M$, где, в частности,

$$
s_{v}=O\left(\frac{1}{M}\right) \text {. }
$$

В $M^{-1 / 2}$-окрестности этой точки, точнее, для точек вида $q_{v} M^{-1 / 2}$, найдем векторное поле сносов, уже с точностью до $M^{-1 / 2}$. Для этого подставим

$$
\frac{n_{v}}{M}=\bar{c}_{v}+x_{v} M^{-1 / 2}
$$

в (25). Выражение, которое при этом получится, аналогично выражению для линеаризованной системы (15). Законы сохранения выполняются и здесь. Поэтому в этом пределе мы получаем марковский процесс с линейным сносом, т. е. процесс Орнштейна-Уленбека, как единственный обратимый стационарный гауссовский процесс.

Матрицу диффузии можно найти из условия стационарности гауссовской меры

$$
L^{*} \rho=0 .
$$

Это условие приводит к уравнению

$$
D \beta=-\lambda,
$$

или

$$
D=-\lambda \beta^{-1}=-\gamma
$$


Иначе говоря, матрица диффузии совпадает с матрицей кинетических коэффициентов. Эта связь между $D$ и $\beta$ имеет место, конечно, и в общей теории равновесных флуктуаций [12]. Матрицу разновременных ковариаций

$$
\phi_{v w}(t)=\left\langle\xi_{v}(t) \xi_{w}(0)\right\rangle, \quad t>0,
$$

можно найти из системы линейных уравнений

$$
\frac{d \phi}{d t}=\lambda \phi
$$

с начальным условием $\phi(0)=\beta^{-1}$. Так что

$$
\phi(t)=e^{\lambda t} \beta^{-1} .
$$

Очевидна симметрия матрицы $\phi$. Действительно, соотношения Онзагера можно записать в виде $\lambda^{\prime}=\beta \lambda \beta^{-1}$, где штрих означает транспонирование. Отсюда

$$
e^{\lambda^{\prime} t}=\beta e^{\lambda t} \beta^{-1}
$$

и, значит, матрица $e^{\lambda t} \beta^{-1}$ симметрична.

Формула Кубо. Рассмотрим матрицу кинетических коэффициентов $\gamma_{u v}=$ $\lambda_{u v} \bar{c}_{v}$. В окрестности неподвижной точки сравним квадратичную форму ковариаций и квадратичную часть энтропии Больцмана. Обе зависят от системы химических реакций и определяются приведением квадратичных форм с помощью законов сохранения.

Если ввести процесс скорости

$$
J_{v}(t)=\frac{d}{d t} \xi_{v}(t)
$$

то его матрица ковариаций

$$
\vartheta_{v w}(t)=\left\langle J_{v}(t) J_{w}(0)\right\rangle
$$

имеет вид

$$
\vartheta(t)=-\frac{d^{2}}{d t^{2}} \phi(t)=-\lambda^{2} \phi(t), \quad t>0 .
$$

Отсюда следует знаменитая формула Кубо (вернее, ее классический вариант)

$$
\int_{0}^{\infty} \vartheta(t) d t=\gamma
$$

где $\gamma$ - матрица кинетических коэффициентов.

3.3. Как получаются необратимые реакции. Проблема необратимости по времени - центральная проблема в физике, и тем более в биологии. Поэтому важно понять, какие вообще могут быть основные элементарные источники необратимости.

Необратимость связана с открытостью системы, в частности с переходами типа транспорта. Например, введением входов, выходов или транспортных 
каналов между реакциями, введением дополнительных веществ. Это может реализоваться физически за счет внешних сил (тяжести, электромагнитных), потока, в котором происходят реакции, или за счет разности в скорости диффузии.

Другой источник необратимости составляют различные аналоги знаменитой идеи Больцмана, что на математическом языке было названо пределом Больцмана-Грэда. Есть много других параметров в замкнутой системе, скейлинг которых и последующий предельный переход позволяют получать необратимые системы. Так, например, большие уклонения в системе можно рассматривать как специальный скейлинг по начальному состоянию, которое уводится далеко от равновесного состояния, после чего изучается, по какому пути оно будет возвращаться к равновесию.

3.3.1. Транспорт. Пусть есть несколько замкнутых систем химических реакций. Можно объединить их в общую систему, допустив перенос (транспорт) вещества из одной системы $i$ в другую $j$. Мы будем говорить при этом, что есть транспортный канал из $i$ в $j$. Мы хотим показать, что при добавлении транспорта система, вообе говоря, становится необратимой.

Пусть задана замкнутая обратимая система химических реакций, удовлетворяющая условию детального баланса (9) с параметрами $b_{v}$ пуассоновской меры. Введем дополнительно входы и выходы, как взаимно обратные реакции, с параметрами $a_{\text {in }, v}, a_{\text {out }, v}$ для некоторых веществ. Чтобы система осталась химически обратимой, эти параметры должны удовлетворять ограничениям

$$
b_{v}=\frac{a_{\text {in }, v}}{a_{\text {out }, v}} .
$$

Если рассмотреть теперь две независимые замкнутые системы химических реакций и соединить их транспортным каналом с интенсивностями

$$
a_{12, v} n_{1, v}, \quad a_{21, v} n_{2, v},
$$

где $n_{i, v}$ - число молекул типа $v$ в системе $i=1,2$, то снова получаем, что условие (9) будет выполняться только для одного значения параметров $a_{12, v} / a_{21, v}$.

3.3.2. Постоянные концентрации. В химический кинетике часто встречается предположение, что одна или более концентраций постоянны. Прежде всего возникает вопрос, когда система реакций, с фиксированными концентрациями некоторых из веществ, необратима.

Рассмотрим обратимую систему $R$ химических реакций с веществами из множества $\mathscr{V}$. Пусть $b_{v}$ - концентрации, удовлетворяющие условию детального баланса. Фиксируем как-то концентрации $c_{v}, v \in \mathscr{W} \subset \mathscr{V}$; вообще говоря, они отличны от $b_{v}$. Рассмотрим редуцированную систему реакций с веществами из множества $\mathscr{V} \backslash \mathscr{W}$, предполагая, что нет реакций, в которых участвовали бы только вещества из $\mathscr{W}$.

Тогда числа $\phi(v)=c_{v} / b_{v}$ должны удовлетворять уравнению

$$
\prod_{v} \phi(v)^{d_{-}(v, r)}=\prod_{v} \phi(v)^{d_{+}(v, r)}
$$


для любой реакции $r \in R$. Таким образом, функция $h(v)=\ln \phi(v)$ является аддитивным первым интегралом любой реакции $r \in R$, т. е.

$$
\sum_{v} d_{-}(v, r) h(v)=\sum_{v} d_{+}(v, r) h(v)
$$

При этом $h(v), v \in \mathscr{W},-$ заданные положительные числа, а остальные $h(v)$, $v \notin \mathscr{W},-$ переменные. Обозначим $\mathscr{L}$ множество всех аддитивных интегралов нашей системы химических реакций, а $\mathscr{H}_{\mathscr{W}}$ - подпространство, состоящее из функций $f(v)$ таких, что $f(v)=\ln \left(c_{v} / b_{v}\right)$ для $v \in \mathscr{W}$. Размерность этого подпространства равна $|\mathscr{V}|-|\mathscr{W}|$. Таким образом, если $|\mathscr{W}|>\operatorname{dim} \mathscr{L}$, то в общем положении (т. е. для открытого всюду плотного множества значений $c_{v}$, $v \in \mathscr{W})$ пересечение $\mathscr{L} \cap \mathscr{H}_{\mathscr{W}}$ пусто. При этом исключительные значения параметров $c_{v}, v \in \mathscr{W}$, представляют собой проекцию подпространства $\mathscr{L}$ на координатную плоскость $\left\{c_{v}, v \in \mathscr{W}\right\}$ и не могут заполнять всю эту плоскость, если $|\mathscr{W}|<\operatorname{dim} \mathscr{L}$. Таким образом, если в обратимой системе $R$ зафиксировано $k+1$, где $k=\operatorname{dim} \mathscr{L}$, то полученная описанным образом система, вообще говоря, не будет обратимой.

ПримеР 9. Для набора реакций с участием атомов С, O, Н углерода, кислорода и водорода имеется три первых интеграла, соответствующих сохранению количества каждого из этих трех атомов. Для достаточно богатого набора химических реакций органической химии других независимых интегралов нет. Таким образом, здесь $k+1=4$.

ЗАмЕчАНИЕ 10. Если имеются реакции с участием только веществ из $W$, то их надо исключить из списка реакций редуцированной системы. Если исключение этих реакций не меняет $\operatorname{dim} \mathscr{L}$ (например, если реакцию $r$ можно заменить на цепочку реакций), то предыдущие выводы сохраняются, так как они основаны только на сравнении $\operatorname{dim} \mathscr{L}$ и $|W|$.

Выше было существенно, что, с точностью до исключения упомянутых выше реакций, условие детального баланса редуцированной системы совпадает с условием детального баланса для исходной системы. Для условия унитарности и неподвижных точек это уже неверно.

Формально, постоянства концентраций, например, одного вещества $v_{0}$ можно добиться, используя различные масштабные пределы. Один из вариантов считать концентрацию $v_{0}$ большой, точнее, в выражение (2) для интенсивностей марковского процесса подставить $M n_{v_{0}}$ вместо $n_{v_{0}}$, или, что то же самое, из $m_{-}(r)$ вычесть $d_{-}\left(v_{0}, r\right)$. Тогда предельные кинетические уравнения

$$
\frac{d c_{v}(t)}{d t}=f\left(c_{1}, \ldots, c_{V}\right)
$$

будут иметь вид

$$
\begin{aligned}
& \frac{d c_{v}(t)}{d t}=f\left(c_{v}, v, c_{v_{0}}\right), \quad v \neq v_{0}, \\
& \frac{d c_{v_{0}}(t)}{d t}=0, \quad c_{v_{0}}(0)=c_{v_{0}} .
\end{aligned}
$$


При другом способе в марковском процессе вводится вход и выход $v_{0}$, который имеет большие скорости $a_{v, \text { in }}, a_{v, \text { out }}$, причем так, чтобы стационарное распределение процесса "вход-выход” имело заданную величину. Очевидно, что в пределе получаем постоянную концентрацию.

Обратная задача. Как получить данную необратимую реакцию из обратимых? Рассмотрим некоторую необратимую систему $R_{n}$, состоящую из нескольких пар взаимно обратных реакций. Они определяются своими скоростями $a_{r}$. Заметим, что для некоторых значений $a_{r}$ эта система реакций обратима. Предположим, что в каждой реакции $r$ участвуют некоторые вещества $w=w(r)$ (энзимы). Тогда в выражении (2) для интенсивностей реакций должен быть множитель $c_{w}$. Если предположить, как выше, что $c_{w(r)}$ постоянны, то скорости $a_{r}$ меняются на $a_{r} c_{w(r)}$, и подбором концентраций $c_{w(r)}$ можно получить нужные значения $a_{r}$.

\section{4. Стохастические локальные модели}

4.1. Стохастические модели со смешанной динамикой. Классическая химическая кинетика является типичным примером того, что в физике называется теорией среднего поля. Возможны также смешанные модели, где реакции описываются моделями среднего поля, но вводится еще локальный транспорт. Эти модели полезны для объединения химической кинетики и химической термодинамики в одну микроскопическую модель. В моделях, введенных в [13], динамика среднего поля для химических реакций дополняется свободным движением частиц. Эти модели позволяют вывести из них основные законы химической термодинамики. Здесь мы приведем только вид предельной динамики, отсылая читателя за всеми ее применениями к [13], [21].

Динамика конечной системы частиц. Определим сначала динамику конечной системы частиц в объеме $\Lambda \subset \mathbb{R}^{3}$ с периодическими граничными условиями. В момент $t=0$ в этот объем равномерно и независимо бросается $N$ частиц, где $N$ случайно, распределено по Пуассону с плотностью $\langle N\rangle / \Lambda=c$. Каждой частице независимо приписывается тип $j$ и скорость $v$, которая имеет плотность $p_{0}(j, v)$ (относительно лебеговой меры), причем

$$
\sum_{j} \int p_{0}(j, v) d v=1 .
$$

Пусть, для простоты, возможны только бинарные реакции

$$
r=(j, v),\left(j_{1}, v_{1}\right) \rightarrow\left(j^{\prime}, v^{\prime}\right),\left(j_{1}^{\prime}, v_{1}^{\prime}\right) .
$$

Если бы скорости принимали конечное число значений, то система химических реакций определялась бы в точности как в разделе 1. В случае непрерывных скоростей вместо параметров $a_{r}$ в (2) мы введем плотности интенсивностей, т. е. интегрируемые (по всем четырем переменным) функции

$$
a_{r}\left(\left(j_{1}, v_{1}\right),\left(j_{1}^{\prime}, v_{1}^{\prime}\right) \mid(j, v),\left(j^{\prime}, v^{\prime}\right)\right) \geqslant 0 .
$$

Таким образом, каждая частица $i$ меняет свой тип и скорость в случайные моменты

$$
t_{i 1}<t_{i 2}<\cdots
$$


В промежутке между этими моментами частица движется с постоянной скоростью, получаемой в последней своей реакции.

Динамика бесконечной системы. Рассмотрим множество $\mathbf{X}$ счетных локально конечных конфигураций $X=\left\{x_{i}, v_{i}, j_{i}\right\}$ частиц в $\mathbb{R}^{3}$, где каждая частица $i$ имеет координату $x_{i}$, скорость $v_{i}$ и тип $j_{i}$. Обозначим $\mathfrak{M}$ множество всех вероятностных мер на $\mathbf{X}$ со следующими свойствами:

(i) координаты этих частиц образуют однородное пуассоновское точечное поле на $\mathbb{R}^{3}$ с некоторой постоянной плотностью $c$;

(ii) векторы $\left(j_{i}, v_{i}\right)$ распределены с общей плотностью $p(j, v)$,

$$
\sum_{j} \int_{\mathbb{R}^{3}} p(j, v) d v=1
$$

и независимы от координат и от других частиц.

Случайная динамика на $\mathbf{X}$ задается парой $\left(\mathbf{X}^{0, \infty}, \mu\right)$, где $\mu=\mu^{0, \infty}-$ вероятностная мера на множестве $\mathbf{X}^{0, \infty}$ счетных наборов $X^{0, \infty}(t)=\left\{x_{i}(t), v_{i}(t), j_{i}(t)\right\}$ кусочно линейных траекторий $\left(x_{i}(t), v_{i}(t), j_{i}(t)\right), 0 \leqslant t<\infty$. При этом мера $\mu$ принадлежит семейству мер $\mathfrak{M}^{0, \infty}$ на $X^{0, \infty}(t)$, определяемому следующими свойствами.

1) Если для любого $t$ обозначить через $\mu(t)$ меру, индуцируемую мерой $\mu$ на $\mathbf{X}$, то $\mu(t) \in \mathfrak{M}$. Обозначим

$$
c_{t}(j, v)=c_{t} p_{t}(j, v)
$$

где $c_{t}, p_{t}$ - концентрация и плотности в момент $t$.

2) Траектории $\left(x_{i}(t), v_{i}(t), j_{i}(t)\right)$ независимы для разных $i$ и каждая из них является траекторией некоторого марковского процесса, не обязательно однородного по времени. Этот процесс определяется начальной мерой $\mu(0) \in \mathfrak{M}$ и инфинитезимальными переходными вероятностями в момент $t$, при этом последние не зависят от координат, скоростей и типов других частиц, но зависят от $c_{t}(j, v)$ в момент $t$.

3) Эволюция пары $(j, v)$ для индивидуальной частицы определяется следующим уравнением Колмогорова:

$$
\frac{\partial p_{t}\left(j_{1}, v_{1}\right)}{\partial t}=\sum_{j} \int\left(P\left(t ; j_{1}, v_{1} \mid j, v\right) p_{t}(j, v)-P\left(t ; j, v \mid j_{1}, v_{1}\right) p_{t}\left(j_{1}, v_{1}\right)\right) d v
$$

где

$$
P\left(t ; j_{1}, v_{1} \mid j, v\right)=\sum_{j^{\prime}, j_{1}^{\prime}} \int 2 a_{r}\left(\left(j_{1}, v_{1}\right),\left(j_{1}^{\prime}, v_{1}^{\prime}\right) \mid(j, v),\left(j^{\prime}, v^{\prime}\right)\right) c_{t}\left(j^{\prime}, v^{\prime}\right) d v^{\prime} d v_{1}^{\prime}
$$

Заметим, что в реакциях могут быть законы сохранения энергии (см. [22], [13]), тогда интегрирование в последней формуле включает соответствующие $\delta$-функции.

4) Координата частицы $i$ изменяется по закону

$$
x_{i}(t)=x_{i}(0)+\int_{0}^{t} v_{i}(s) d s .
$$


Пусть задана мера $\mu(0) \in \mathfrak{M}$. Рассмотрим теперь последовательность конечных систем частиц, определенных выше, в расширяющейся системе конечных объемов $\Lambda \uparrow \mathbb{R}^{3}$. Начальная мера для каждой из них является ограничением меры $\mu(0)$ на соответствующий объем $\Lambda$. Поэтому в начальный момент мы можем фиксировать отдельную частицу $i$ в $\mathbb{R}^{3}$ и говорить о ее траекториях $x_{i}^{(\Lambda)}(t)$ в каждом $\Lambda$, начиная с некоторого. Кроме того, определена ее траектории $x_{i}(t) \in \mathbf{X}^{0, \infty}$ в $\mathbb{R}^{3}$.

Мы видим, что два определения - систем конечного и бесконечного числа частиц - весьма различны. Следующий результат показывает связи между ними.

ТЕОрема 11. Для любых $i$ u $t$ имеет место сходимость по вероятности

$$
\lim _{\Lambda \uparrow \mathbb{R}^{3}} x_{i}^{(\Lambda)}(t)=x_{i}(t) .
$$

Доказательство см. в [13; дополнение].

\section{2. Уравнение Больцмана с химическими реакциями.}

4.2.1. Модель и результат. Пусть в момент $t=0$ в кубе $\Lambda \subset \mathbb{R}^{d}$ имеется $N<\infty$ частиц, каждая из которых характеризуется координатой $x \in \Lambda$, скоростью $v \in \mathbb{R}^{d}$ и типом $q \in\{1, \ldots, Q\}$. Начальный вектор $\left(x_{i}(0), q_{i}(0), v_{i}(0)\right.$ : $i=1, \ldots, N)$ обозначается $\omega_{0}^{\Lambda}$. Предположим, что начальная мера $\mu_{\Lambda}$ на множестве конфигураций $\omega_{0}^{\Lambda}$ в $\Lambda$ определена вероятностями $p_{N}$ того, что число частиц в $\Lambda$ равно $N$, и условными плотностями (при данном $N$ )

$$
\rho_{N, \Lambda}\left(x_{1}, v_{1}, q_{1}, \ldots, x_{N}, v_{N}, q_{N}\right)<C^{N},
$$

симметричными относительно группы перестановок $S_{N}$ и нормированными так, что

$$
\frac{1}{N !} \sum_{q_{1}, \ldots, q_{N}} \int \rho_{N, \Lambda} \prod_{k=1}^{N} d x_{k} d v_{k}=1
$$

Тогда $k$-частичные корреляционные функции определяются следующим образом:

$$
f_{k}\left(x_{1}, v_{1}, q_{1}, \ldots, x_{k}, v_{k}, q_{k}\right)=\sum_{N=k}^{\infty} \frac{p_{N}}{(N-k) !} \sum_{q_{k+1}, \ldots, q_{N}} \int \rho_{N, \Lambda} \prod_{j=k+1}^{N} d x_{j} d v_{j} .
$$

Поэтому вероятность того, что в каждом из малых объемов $d x_{i} d v_{i}, i=1, \ldots, k$, есть частица типа $q_{i}$, равна

$$
f_{k}\left(x_{1}, v_{1}, q_{1}, \ldots, x_{k}, v_{k}, q_{k}\right) \prod_{i=1}^{k} d x_{i} d v_{i}
$$

Для каждой начальной конфигурации $\omega_{0}^{\Lambda}$ определим марковский процесс с непрерывным временем

$$
\xi(t, \omega)=\xi_{N, \Lambda}\left(t, \omega, \omega_{0}^{\Lambda}\right)=\left(x_{i}(t), v_{i}(t), q_{i}(t): i=1, \ldots, N\right) .
$$


В этой системе аргумент $\omega_{0}^{\Lambda}$ отвечает случайности, связанной с начальной (при $t=0)$ конфигурацией, а аргумент $\omega$ отвечает случайности, связанной со случайными скачками. Эвристически $\xi(t, \omega)$ определяется как смесь детерминированной (кусочно линейной) динамики (с периодическими граничными условиями) для координат и случайных скачков для скоростей и типов. Эти случайные скачки предполагаются бинарными реакциями, меняющими скорости и типы. Точнее, скорости и типы предполагаются кусочно постоянными на $[0, \infty)$; скачки происходят в случайные моменты времени

$$
0<t_{1}(\omega)<\cdots<t_{k}(\omega)<\cdots .
$$

На интервалах времени $t \in\left(t_{i}, t_{i+1}\right]$ частицы движутся свободно, т. е. с постоянными скоростями $v_{k}(t)=v_{k}\left(t_{i}+0\right)$. Таким образом, почти всюду $v_{k}(t)=\frac{d x_{k}(t)}{d t}$. Другими словами, координаты в момент $t \in \mathbb{R}_{+}$определены как

$$
x_{i}(t, \omega)=x_{i}(0)+\int_{0}^{t} v_{i}(t, \omega) d t .
$$

В то же время любые две частицы $i, j$ (независимо от других пар) на любом интервале времени $(t, t+d t)$ меняют свои типы и скорости с интенсивностями (плотностями)

$$
\lambda\left(q_{i}^{\prime}, v_{i}^{\prime}, q_{j}^{\prime}, v_{j}^{\prime} \mid x_{i}(t), v_{i}(t), q_{i}(t), x_{j}(t), v_{j}(t), q_{j}(t)\right) d t .
$$

Функции $\lambda$ предполагаются неотрицательными, ограниченными, гладкими, трансляционно инвариантными и равными нулю, если выполнено хотя бы одно из следующих условий:

1) $\left|x_{i}-x_{j}\right| \geqslant 2 r$

2) для некоторого $v^{0}>0$ либо $\left|v_{i}^{\prime}\right|>v^{0}$, либо $\left|v_{j}^{\prime}\right|>v^{0}$.

Эти скачки не меняют координат, но меняют скорости и типы:

$$
\left(q_{i}, v_{i}, q_{j}, v_{j}\right)=\left(q_{i}, v_{i}, q_{j}, v_{j}\right)(t) \rightarrow\left(q_{i}^{\prime}, v_{i}^{\prime}, q_{j}^{\prime}, v_{j}^{\prime}\right)=\left(q_{i}^{\prime}, v_{i}^{\prime}, q_{j}^{\prime}, v_{j}^{\prime}\right)(t+0) .
$$

Обозначим

$$
B\left(v, q, v^{\prime}, q^{\prime} \mid v_{1}, q_{1}, v_{2}, q_{2}\right)=\lambda\left(v, q, v^{\prime}, q^{\prime} \mid x, v_{1}, q_{1}, x, v_{2}, q_{2}\right) .
$$

Таким образом, для данных $N, \Lambda, \lambda, r$ мы задали семейство $\xi_{N, \Lambda}(t)$ процессов с конечным числом частиц.

ЗАмЕчАниЕ 12. Эта модель допускает множество обобщений, например когда движение частиц между скачками определяется гамильтоновой системой с парным потенциалом $V(x-y)$ с радиусом взаимодействия $r$ :

$$
\frac{d^{2} x_{i}(t)}{d t^{2}}=-\sum_{j: j \neq i} \frac{\partial V\left(x_{i}-x_{j}\right)}{\partial x_{i}} .
$$

Начальные условия. Мы рассматриваем семейство $\mu_{r}$ начальных распределений в конечном объеме $\Lambda$ или в $\mathbb{R}^{3}$ с корреляционными функциями $f_{k}^{(r)}\left(0 ; x_{1}, v_{1}, q_{1}, \ldots, x_{k}, v_{k}, q_{k}\right)$, параметризованными положительными числами $r$ (радиусами взаимодействия). Предполагается, что это семейство удовлетворяет следующим условиям. 
1. Скейлинг Больцмана-Грэда: для некоторой фиксированной ограниченной неотрицательной функции $f(x, v, q)$

$$
f_{1}^{(r)}(0 ; x, v, q)=r^{-d+1} f(x, v, q) .
$$

Этот скейлинг означает, в частности, что средняя плотность числа частиц растет как $r^{-d+1}$ при $r \rightarrow 0$. Заметим, что в это же время "эффективный” объем $N r^{d}$ ( $r$ можно рассматривать как “эффективный” радиус частиц), занятый частицами, стремится к нулю как $r$.

2. Экспоненциальное убывание корреляций:

$$
\begin{aligned}
& \left|f_{2}^{(r)}\left(0 ; x_{1}, v_{1}, q_{1}, x_{2}, v_{2}, q_{2}\right)-f_{1}^{(r)}\left(0 ; x_{1}, v_{1}, q_{1}\right) f_{1}^{(r)}\left(0 ; x_{2}, v_{2}, q_{2}\right)\right| \\
& \quad<C \exp \left(-C_{1} r^{-1}\left|x_{1}-x_{2}\right|\right)
\end{aligned}
$$

для всех положительных достаточно малых $r$.

3. Сильная устойчивость: число частиц $n(A)$ в произвольном объеме $A \subset \Lambda$ равномерно ограничено п.н. величиной $c r^{-d+1}|A|$ для некоторого $c>0$. Физически это естественное условие.

4. Оценки сверху: для некоторого $C>0$

$$
f_{k}^{(r)}\left(0 ; x_{1}, v_{1}, q_{1}, \ldots, x_{k}, v_{k}, q_{k}\right)<C^{k} r^{(-d+1) k}, \quad k \geqslant 1 .
$$

Приведем пример точечного поля, удовлетворяющего условиям 1-4. Рассмотрим сначала пуассоновское поле с корреляционными функциями

$$
g_{k}\left(x_{1}, v_{1}, q_{1}, \ldots, x_{k}, v_{k}, q_{k}\right)=\prod_{i=1}^{k} g_{1}\left(x_{i}, v_{i}, q_{i}\right)
$$

для некоторой гладкой ограниченной функций $g_{1}$. Определим тогда $\mu_{r}$ как точечное поле, полученное из пуассоновского поля с $k$-частичными функциями $g_{k}^{(r)}=r^{(1-d) k} g_{k}$ преобразованием $F$ на множестве конфигураций, где конфигурация $\omega_{1}=F\left(\omega_{0}\right)$ получается из $\omega_{0}$ удалением любой частицы, для которой в $\omega_{0}$ есть другая частица на расстоянии меньшем или равном $2 r$. Тогда свойства 1 и 3 следуют из определения. Свойство 4 следует из монотонности

$$
f_{k}^{(r)} \leqslant g_{k}^{(r)}
$$

Свойство 2 следует из стандартных оценок вероятности того, что существует кластер (последовательность частиц с расстоянием не более $2 r$ каждой от предыдущей) диаметра $\left|x_{1}-x_{2}\right| / 2$, содержащий по крайней мере одну из частиц $x_{1}$ или $x_{2}$.

4.2.2. Существование кластерной динамики. Мы скажем, что две частицы $i, j$ взаимодействуют в момент $t$, если в этот момент между ними произошла реакция. Тогда в этот момент они находились на расстоянии не большем $2 r$. Обозначим $s_{i j}$ первый момент взаимодействия частиц $i$ и $j$. Рассмотрим следующие конечные случайные графы $G^{\Lambda}=G^{\Lambda}(\tau)=G^{\Lambda}(\tau, \omega)$. Их вершинами являются частицы, нумеровать частицы удобно их начальными векторами $x_{i}(0)$. Две вершины связываются ребром, если в какой-то момент из интервала $[0, \tau]$ они взаимодействовали. При фиксированных $\tau$ и $\Lambda$ множество 
вершин произвольной связной компоненты графа $G^{\Lambda}=G^{\Lambda}(\tau)$ называется динамическим кластером (в $\Lambda$ ).

Если в точке $x$ в начальный момент есть частица, то обозначим $P_{k}^{\Lambda}(\tau \mid x)$ условную вероятность того, что содержащий ее динамический кластер состоит из ровно $k$ частиц. Обозначим

$$
\rho=\max _{x} \sum_{q} \int f_{1}(x, v, q) d v .
$$

Тогда имеет место следующая экспоненциальная оценка (см. [23]).

Теорема 13. Существуют константы $C>0 u \alpha_{0}>0$ такие, что для произвольных $\tau, v^{0}, r u$

$$
\rho=\alpha\left(\tau v^{0} r^{d-1}\right)^{-1}
$$

с произвольной $0<\alpha<\alpha_{0}$ равномерно по $k, \Lambda, x$ имеет место неравенство

$$
P_{k}^{\Lambda}(\tau \mid x) \leqslant(C \alpha)^{k-1} .
$$

Напомним, что смысл выражения для плотности состоит в том, что среднее число частиц $\langle N\rangle$ в единичном объеме, умноженное на максимальный объем трубки, заметаемой частицей (т. е. $2 r$-окрестности ее траектории), было меньше некоторого $\alpha_{0}$. Доказательство см. в [23].

Из этой теоремы следует, что с вероятностью 1 любая частица на интервале времени $[0, \tau]$ принадлежит конечному кластеру, зависящему от выбранной частицы и от начальной конфигурации. Более того, существует термодинамический предел, который является кластерной динамикой в $\mathbb{R}^{d}$. Иначе говоря, динамика бесконечного числа частиц складывается из бесконечного числа независимых динамик конечного числа частиц.

Более точно, имеет место следующее кластерное представление динамики. Рассмотрим марковский процесс $\xi_{k}(t)=\left(x_{1}(t), v_{1}(t), q_{1}(t), \ldots, x_{k}(t), v_{k}(t), q_{k}(t)\right)$ на интервале $[0, \tau]$ с $k$ частицами (и при отсутствии других частиц), находящимися в начальный момент в точках $\xi_{k}=\xi_{k}(0)=\left(x_{1}, v_{1}, q_{1}, \ldots, x_{k}, v_{k}, q_{k}\right)$. Будем обозначать $\gamma=\gamma(\eta \mid \xi)$ траекторию процесса $\xi_{k}(t)$, начинающуюся в момент 0 в точке $\xi$ и кончающуюся в момент $\tau$ в точке $\eta=\left(x_{1}^{\prime}, v_{1}^{\prime}, q_{1}^{\prime}, \ldots, x_{k}^{\prime}, v_{k}^{\prime}, q_{k}^{\prime}\right)$. Обозначим $P^{\tau}(\gamma(\eta \mid \xi))$ условное распределение на множестве $\{\gamma(\eta \mid \xi)\}$ таких траекторий. Пусть $\Gamma_{k}=\Gamma_{k}(\eta \mid \xi)$ - множество всех траекторий $\gamma$, которые образуют $k$-кластер.

Тогда, используя (28), получаем

$$
\begin{aligned}
f(\tau ; x, v, q)=\sum_{k=1}^{\infty} & \sum_{q_{1}, \ldots, q_{k}}^{\infty} \int_{R^{d k} \times \Lambda^{k}} \prod_{i=1}^{k} d x_{i} d v_{i} \prod_{i=2}^{k} d x_{i}^{\prime} d v_{i}^{\prime} \\
& \times \int_{\Gamma_{k}} Q(\gamma) d P^{\tau}\left(\gamma\left(\left(x, v, q, x_{2}^{\prime}, v_{2}^{\prime}, q_{2}^{\prime}, \ldots, x_{k}^{\prime}, v_{k}^{\prime}, q_{k}^{\prime}\right) \mid \xi\right)\right) \\
& \times f_{k}\left(0 ; x_{1}, v_{1}, q_{1}, \ldots, x_{k}, v_{k}, q_{k}\right),
\end{aligned}
$$

где $Q(\gamma)=Q(\gamma(\eta \mid \xi))$ - условная вероятность того, что другие частицы конфигурации $\omega_{0}^{\Lambda}$ не взаимодействуют с выделенными $k$ частицами. 
4.2.3. Вывод уравнения Больцмана. Если кластерная динамика существует для фиксированных $r$, то уравнение Больцмана имеет место только в пределе Больцмана-Грэда. Зафиксируем $\tau$ и $v^{0}$ и предположим, что функция $f(x, v, q)$ из условия (30) достаточно мала, чтобы было выполнено условие (31). Тогда имеет место следующее утверждение.

Теорема 14. В пределе Больимана-Грэда для любого $t<\tau$ существуют функции плотности

$$
\lim _{r \rightarrow 0} r^{d-1} f^{(r)}(t ; x, v, q)=f(t ; x, v, q),
$$

которые удовлетворяют уравнению Больцмана

$$
\begin{aligned}
\frac{\partial f}{\partial t}(x, v, q)=- & v \frac{\partial f}{\partial x}(x, v, q) \\
& +\sum_{q^{\prime}, q_{1}, q_{2}} \int\left[B\left(v, q, v^{\prime}, q^{\prime} \mid v_{1}, q_{1}, v_{2}, q_{2}\right) f\left(x, v_{1}, q_{1}\right) f\left(x, v_{2}, q_{2}\right)\right. \\
& \left.-B\left(v_{1}, q_{1}, v_{2}, q_{2} \mid v, q, v^{\prime}, q^{\prime}\right) f(x, v, q) f\left(x, v^{\prime}, q^{\prime}\right)\right] d v_{1} d v_{2} d v^{\prime}
\end{aligned}
$$

В нулевом приближении (если нет взаимодействия) есть только 1-кластеры, и тогда

$$
f(t+\delta ; x, v, q)=f(t ; x-v \delta, v, q) .
$$

Вычитая $f(t ; x, v, q)$ из обеих частей этого равенства, деля на $\delta$ и переходя к пределу $\delta \rightarrow 0$, получаем

$$
\frac{\partial f}{\partial t}=-v \frac{\partial f}{\partial x}
$$

В общем случае равенство (32) соответствует событию, что та частица, которая была в точке $x, v$ в момент $t$, не испытала реакций в интервале времени $(t, t+d t)$.

В нашем случае имеет место равенство

$$
f(t+\delta ; x, v, q)=f(t ; x-v \delta, v, q)-A_{1}^{(r)}+B_{1}^{(r)}+O\left(\delta^{2}\right) .
$$

Член $A_{1}^{(r)}$ получается из событий, когда в момент $t$ была частица с параметрами $x-v \delta, v, q$, которая затем (в некоторый момент $s, t<s<t+\delta$ ) испытала реакцию с другой частицей, которая в момент $t$ имела параметры $x_{1}, v_{1}, q_{1}$. Иначе говоря,

$$
\begin{aligned}
A_{1}^{(r)}= & \sum_{q_{1}, q^{\prime}, q_{1}^{\prime}} \int_{t}^{t+\delta} d s \int \lambda\left(q^{\prime}, v^{\prime}, q_{1}^{\prime}, v_{1}^{\prime} \mid x(s), v(s), q(s), x_{1}(s), v_{1}(s), q_{1}(s)\right) \\
& \times f_{2}^{(r)}\left(t ; x-v \delta, v, q ; x_{1}, v_{1}, q_{1}\right) d x_{1} d v_{1} d v^{\prime} d v_{1}^{\prime} .
\end{aligned}
$$

Заметим, что, как следует из кластерного свойства, убывание корреляций сохраняется для любого момента времени в интервале $0<t \leqslant \tau$. Тогда

$$
A_{1}^{(r)} \rightarrow \delta \sum_{q_{1}, q^{\prime}, q_{1}^{\prime}} \int B\left(q^{\prime}, v^{\prime}, q_{1}^{\prime}, v_{1}^{\prime} \mid v, q, v_{1}, q_{1}\right) f_{1}(q, v) f_{1}\left(q_{1}, v_{1}\right) d v^{\prime} d v_{1}^{\prime} d v_{1}
$$


Член $B_{1}^{(r)}$ возникает из событий, когда в момент $t$ имеются две частицы с параметрами $x_{1}, v_{1}, q_{1}, x_{2}, v_{2}, q_{2}$, которые реагируют в момент $s, t<s<t+\delta$, так, что одна из возникающих частиц имеет параметры $x, v, q$. Так что

$$
\begin{gathered}
B_{1}^{(r)}=\sum_{q_{1}, q_{2}, q^{\prime}} \int_{t}^{t+\delta} d s \int \lambda\left(v, q, q^{\prime}, v^{\prime} \mid x_{1}, v_{1}, q_{1}, x_{2}, v_{2}, q_{2}\right) \\
\quad \times f_{2}^{(r)}\left(x_{1}, v_{1}, q_{1}, x_{2}, v_{2}, q_{2}\right) d x_{1} d v_{1} d x_{2} d v_{2} d v^{\prime} .
\end{gathered}
$$

Аналогично (34) имеем

$$
\lim _{r \rightarrow 0} B_{1}^{(r)}=\delta \sum_{q_{1}, q_{2}, q^{\prime}} \int B\left(v, q, q^{\prime}, v^{\prime} \mid v_{1}, q_{1}, v_{2}, q_{2}\right) f_{1}\left(q_{1}, v_{1}\right) f_{1}\left(q_{2}, v_{2}\right) d v_{1} d v_{2} d v^{\prime}
$$

Из кластерного представления динамики легко следует, что остальные кластеры не дают вклада в уравнение Больцмана, так как имеют порядок $O\left(\delta^{2}\right)$.

ЗАмЕчАниЕ 15. Другие модели и другую технику вывода уравнения Больцмана см. в [24]-[31].

4.3. Простейшие модели с транспортом на решетке. Пусть в каждой точке $x$ решетки $\mathbb{Z}^{d}$ может находиться $n_{v}(x)$ частиц типа $v=1, \ldots, V$. В каждой точке задан марковский процесс $\xi_{x}=\left(n_{1}(x), \ldots, n_{V}(x)\right)$ - система химических реакций в смысле раздела 2. Эти процессы независимы и одинаково распределены. В генератор этого процесса добавляются члены, соответствующие простому независимому блужданию с непрерывным временем каждой из частиц, однородному по времени и по пространству. Параметры $\lambda_{e, v}-$ интенсивности скачков, где $e$ пробегает $2 d$ единичных векторов вдоль осей, могут зависеть от типа $v$. Предположим сначала, что вектора сноса

$$
m_{v}=\sum_{e} e \lambda_{e, v} \neq 0
$$

для всех $v$. Мы будем использовать скейлинг

$$
x=\frac{X}{\varepsilon}, \quad t=\frac{\tau}{\varepsilon}, \quad \lambda_{r}(n) \rightarrow \varepsilon \lambda_{r}(n),
$$

где $X \in \mathbb{R}^{d}, \tau \in \mathbb{R}$ - макропеременные. При этом в определении (2) мы полагаем $M=1$, так что число частиц в любой точке имеет порядок $O(1)$. Этот скейлинг означает, в частности, что за конечное макровремя $\tau$ в данной точке происходит $O(\tau)$ реакций.

ТЕОРемА 16. Пусть в начальный момент $t=0$ задано начальное распределение Пуассона

$$
\prod_{x} \prod_{v} \frac{\left(b_{v, x}\right)^{n_{v}(x)}}{n_{v}(x) !} \exp \left(-b_{v, x}\right)
$$

частии, на решетке такое, что $b_{v, x}=c_{v}(0, \varepsilon x)$ для некоторьх гладких ограниченных функиий $c_{v}(0, X), X \in \mathbb{R}^{d}$. Тогда при $\varepsilon \rightarrow 0$ для любой функиии $x(\varepsilon): \mathbb{R}_{+} \rightarrow \mathbb{Z}^{d}$ такой, что $\varepsilon x(\varepsilon) \rightarrow X$, существуют предель концентраций

$$
c_{v}(\tau, X)=\lim _{\varepsilon \rightarrow 0}\left\langle n_{v}\left(\frac{\tau}{\varepsilon}, x(\varepsilon)\right)\right\rangle,
$$


которые удовлетворяют уравнениям

$$
\frac{\partial c_{v}}{\partial \tau}=-m_{v} \frac{\partial c_{v}}{\partial X}+F_{v}\left(c_{1}, \ldots, c_{V}\right)
$$

где функции $F_{v}$ такие же, как правые части уравнения (3).

Вкратце, идеи доказательства таковы. Прежде всего известно и легко доказать, что при отсутствии реакций независимая система частиц удовлетворяет уравнению (35) без последнего члена. Реакции же идут существенно медленнее транспорта, и за конечное макровремя их число в каждой точке $O(1)$. Поэтому в промежутках между реакциями распределения разных типов частиц в каждой точке стремятся быть независимыми и распределенными по Пуассону с некоторыми интенсивностями $c_{v, x}$, благодаря быстрому перемешиванию при блуждании. Поэтому в пределе $\varepsilon \rightarrow 0$ для любого целого $k>0$

$$
\left\langle n_{v, x}\left(n_{v, x}-1\right) \cdots\left(n_{v, x}-k+1\right)\right\rangle_{\text {Poisson }}=c_{v, x}^{k} .
$$

Доказательства существования предельных переходов основаны на том или ином варианте кластерных разложений. Более подробно об этом см. книгу [25; c. 308-313, 315-316] и библиографию там же.

Если все сносы $m_{v}$ равны 0 , то нужен другой (диффузионный) скейлинг

$$
x=\frac{y}{\varepsilon}, \quad t=\frac{\tau}{\varepsilon^{2}}, \quad \lambda_{r}(n) \rightarrow \varepsilon^{2} \lambda_{r}(n),
$$

который отвечает разнице в шкалах времен реакции и теплового движения. При этом возникают уравнения реакции-диффузии: если мы принимаем интенсивности $\lambda_{v}$ скачков в каждом направлении равными $1 / 2$, то

$$
\frac{\partial c_{v}}{\partial \tau}=\frac{1}{2} \Delta c_{v}+F_{v}\left(c_{1}, \ldots, c_{V}\right) .
$$

Идеи доказательства такие же, как для предыдущей теоремы.

Интересен случай, когда координаты сноса являются ненулевыми по одним направлениям (поток) и нулевыми по перпендикулярным (диффузия). Рассмотрим, например, двумерную решетку $\mathbb{Z}^{2}=\{(x, y)\}$ с несколькими типами частиц, где $m_{v, x}=0, m_{v, y} \neq 0$ для всех $v$. Тогда при скейлинге

$$
x=\frac{X}{\varepsilon}, \quad y=\frac{Y}{\varepsilon^{2}}, \quad t=\frac{\tau}{\varepsilon^{2}}, \quad \lambda_{r}(n) \rightarrow \varepsilon^{2} \lambda_{r}(n)
$$

предельные уравнения будут иметь вид

$$
\frac{\partial c_{v}}{\partial \tau}=-m_{v, X} \frac{\partial c_{v}}{\partial Y}+\frac{1}{2} \frac{\partial^{2} c_{v}}{\partial X^{2}}+F_{v}\left(c_{1}, \ldots, c_{V}\right) .
$$

Все вышеприведенные кинетические уравнения могут иметь несколько инвариантных мер, если соответствующие уравнения

$$
F_{v}\left(c_{1}, \ldots, c_{V}\right)=0
$$

имеют несколько неподвижных точек.

Об уравнениях реакции-диффузии см. также [32]-[34]. 


\section{5. Химическая реакция как процесс}

5.1. Обратимость во времени в физике. Отображение (функция, оператор) $f$ из множества $A$ на множество $B$ называется обратимым, если оно взаимно однозначно. При этом $f$ и $g=f^{-1}$ называются взаимно обратными. Если $A=B$, то $f$ называется автоморфизмом.

Автоморфизм $U$ порождает группу автоморфизмов $U^{t}$, где $t \in \mathbb{Z}$, а часто его можно вложить в непрерывную группу с $t \in \mathbb{R}$. Группа автоморфизмов множества $A$ называется обратимой во времени относительно автоморфизма $W$ множества $A$, если

$$
W^{-1} U^{t} W U^{t}=1
$$

или $U^{-t}=W^{-1} U^{t} W$.

В физике понятие обратимости во времени связывается с конкретными автоморфизмами (обычно инволюциями) $W$. Так, в классической ньютоновской физике $n$ частиц рассматриваются группы автоморфизмов многообразия

$$
\Lambda^{n} \times \mathbb{R}^{n d}=\left\{(\bar{x}, \bar{p})=\left(x_{1}, \ldots, x_{n}, p_{1}, \ldots, p_{n}\right), x_{i} \in \Lambda \subset \mathbb{R}^{d}, p_{i} \in \mathbb{R}^{d}\right\}
$$

и $W$ берется равным

$$
W(\bar{x}, \bar{p})=(\bar{x},-\bar{p}) .
$$

В нерелятивистской квантовой механике $A$ является комплексным гильбертовым пространством, группа автоморфизмов - унитарная группа $U^{t}=\exp (i t H)$ с генератором $H$, а $W$ - антилинейное отображение. Например, $w$ антилинейно, если в некотором представлении имеет место равенство

$$
W \phi=\bar{\phi} .
$$

Если $H W=W H$, то выполнено (36).

В релятивистской квантовой теории обратимость во времени может иметь место для одних операторов $W$, и не иметь места для других, как при нарушении четности.

Из этой обратимости при определенных условиях вытекает обратимость соответствующих стохастических систем. Примерами могут служить обратимость трансфер-матрицы в евклидовом подходе к квантовой теории поля, сама матрица рассеяния, а также марковские процессы, получающиеся в пределе слабого взаимодействия.

Матрица рассеяния $S: \mathscr{F} \rightarrow \mathscr{F}$ является унитарным оператором в пространстве $Ф$ ока $\mathscr{F}=\mathscr{F}(\mathscr{H})$, где $\mathscr{H}$ - прямая сумма всех одночастичных подпространств $\mathscr{H}_{q}$ в $\mathscr{F}(\mathscr{H})$. Одночастичное пространство соответствует типу $q$ частицы. Пусть $e_{q, k}-$ ортонормированный базис в $\mathscr{H}_{q}$, где индекс $k$ соответствует импульсам. Пусть $\gamma=\left\{\left(q_{1}, k_{1}\right), \ldots,\left(q_{n}, k_{n}\right)\right\}$, рассмотрим базис

$$
e_{\gamma}=e_{q_{1} k_{1}} \otimes \cdots \otimes e_{q_{n} k_{n}}, \quad n=0,1,2, \ldots,
$$

в $\mathscr{F}$. Обозначим квадраты $\left|S\left(\gamma \rightarrow \gamma^{\prime}\right)\right|^{2}$ матричных элементов $S$ в этом базисе через $w\left(\gamma \rightarrow \gamma^{\prime}\right)$. Тогда из сохранения нормы следует, что

$$
\sum_{\gamma^{\prime}} w\left(\gamma \rightarrow \gamma^{\prime}\right)=\sum_{\gamma^{\prime}} w\left(\gamma^{\prime} \rightarrow \gamma\right)=1,
$$

т. е. что матрица $W=\left(w\left(\gamma \rightarrow \gamma^{\prime}\right)\right)$ дважды стохастическая. 
Заметим, что условие (37) соответствует условию унитарности, введенному в разделе 2. Интуитивно, чтобы из унитарной матрицы рассеяния получить стохастическую динамику, надо поступить так: ввести случайные времена ожидания в состояниях $\gamma$, тогда матрица $W$ будет играть роль матрицы условных вероятностей (в момент окончания ожидания) скачков из $\gamma$ в $\gamma^{\prime}$. Есть еще одна трудность - $\gamma$ зависят от индексов $k$, которые соответствуют импульсам или скоростям. Хотелось бы, чтобы марковским был процесс, получаемый ограничением на множество типов $\{q\}$. И более того, чтобы матрица $W^{0}$, которая получается ограничением матрицы $W$ на множество типов, была также дважды стохастической. Это означает, что происходит восстановление классической химической кинетики, которая обычно определена лишь на типах без учета скоростей. В [21] показано что при некоторых условиях это действительно будет иметь место.

При определенных предельных переходах унитарная квантовая динамика переходит в стохастическую, например в пределе слабого взаимодействия, (см. [35]). При этом в пределе получается обратимый марковский процесс.

5.2. Классическое гамильтоново рассеяние. Первый вопрос состоит в том, может ли вообще связанное состояние быть получено динамически путем столкновения составляющих его частиц. Это, конечно, не всегда возможно. Проще всего это уяснить на классической теории рассеяния, уже на простейшем примере рассеяния одной частицы на центре (см. [36]). Основным ограничением является, конечно, закон сохранения энергии, т. е. суммы внутренних энергий связанных состояний и кинетических энергий. Неизвестно, единственное ли это ограничение.

Рассеяние частицы на внешнем потенциале. Рассмотрим одномерную задачу рассеяния классической частицы на гладком внешнем потенциале $V(x)$, $x \in \mathbb{R}$, равном нулю вне некоторого ограниченного множества. Пусть частица движется из $-\infty$ со скоростью $v_{0}$. Известно, что образование связанного состояния, т. е. захват частицы потенциалом, возможно только для множества скоростей меры нуль. Это следует из обратимости динамики по времени. Действительно, возможны три случая:

1) частица изменяет направление движения; это может быть лишь в точке, где $\frac{d V}{d x} \neq 0$; тогда частица проделывает путь в обратном направлении;

2) частица останавливается; это может быть лишь в точках, где $\frac{d V}{d x}=0$; понятно, что множество таких значений $v_{0}$ конечно;

3) частица проходит в $+\infty$; тогда она нигде не останавливается и не поворачивает обратно.

Для рассеяния частицы на внешнем потенциале в размерности $d \geqslant 2$ есть аналогичные результаты (см. [36]).

Если, однако, ввести возможность мгновенной или быстрой диссипации энергии, передачи части энергии некоей третьей частице или перехода части энергии во внутреннюю энергию каких-то степеней свободы комплекса из двух частиц, то образование связанного состояния возможно. Тогда частица может застрять в потенциальной яме, образуя связанное состояние. 
Двухчастичное и трехчастичное рассеяние. Двухчастичное рассеяние сводится к предыдущему случаю, а для трехчастичного имеются примеры из небесной механики (см. [37]), которые показывают, какие реакции в этом случае возможны.

5.3. Скорости реакций из локальной теории. Следующая простая модель показывает, как можно получать интенсивности реакций из локальных моделей.

Рассмотрим равновесную свободную систему из $N_{i}, i=1,2$, шариков радиусов $r_{i}$ в объеме $M$, которые движутся, не замечая друг друга, с максвелловским распределением скоростей. Обозначим $c_{i}=N_{i} / M$ удельное число этих частиц. Легко доказать, что число $R(N, M, t)$ бинарных "столкновений-перекрытий" шариков разных типов на большом промежутке времени $[0, t]$ асимптотически (при $\left.N_{i}, M \rightarrow \infty\right)$ равно

$$
R(N, M, t) \sim t N_{1} N_{2} \frac{4 \pi\left(r_{1}+r_{2}\right)^{3}}{3 M} .
$$

Значит, среднее число столкновений в единице времени и объема равно

$$
\frac{R}{t M}=\frac{4 \pi\left(r_{1}+r_{2}\right)^{3}}{3} c_{1} c_{2} .
$$

При этом доля энергетичных столкновений, т. е. таких, что сумма кинетических энергий не менее некоторого числа $T_{\mathrm{cr}}$, составляет примерно

$$
a_{r} \exp \left(-\beta T_{\mathrm{cr}}\right) c_{1} c_{2}
$$

для некоторой константы $a_{r}$, соответствующей данной реакции. Это есть закон Аррениуса.

Различные математические модели, связанные с переходом химической энергии в кинетическую, можно найти в [22], [13], [21].

5.4. Динамика с немгновенными реакциями. Рассмотрим бесконечную систему точечных частиц в $\mathbb{R}^{d}$, где каждая частица определяется координатой $x$, скоростью $v$ и типом $j=1, \ldots, J$. Частицы взаимодействуют посредством двухчастичных потенциалов $U_{j j^{\prime}}\left(x-x^{\prime}\right)$. Предполагается, что радиусы взаимодействия $R_{j j^{\prime}}$ конечны. Определим граф с вершинами в координатах частиц, соединяя две частицы типов $j, j^{\prime}$ ребром, если расстояние между ними не превосходит $R_{j j^{\prime}}$. Вершине приписывается, кроме того, скорость и тип соответствующей частицы. Рассмотрим связные конечные маркированные графы $G$ с отмеченными таким образом вершинами. Таких графов континуум, и мы введем классы их эквивалентности. Два таких графа $G_{1}$ и $G_{2}$ назовем эквивалентными, если выполнено хотя бы одно из следующих трех условий:

1) $G_{1}$ и $G_{2}$ изоморфны как маркированные графы, т. е. они изоморфны как графы и марки соответствующих вершин совпадают;

2) $G_{1}$ и $G_{2}$ получаются один из другого сдвигом всех координат на один и тот же вектор;

3) $G_{1}$ и $G_{2}$ получаются один из другого гамильтоновой динамикой соответствующей конечной системы частиц с потенциалами $U_{j j^{\prime}}\left(x-x^{\prime}\right)$ за конечное время. 
Класс эквивалентности назовем кластером или метастабильной частицей. Метастабильная частица называется устойчивой частицей или связанным состоянием, если она, в отсутствие других частиц, существует бесконечное время.

Бесконечная система частиц в $\mathbb{R}^{d}$ в любой момент времени разбивается на (максимальные) связные компоненты. Каждая соответствует определенному кластеру.

Предположим теперь, что существует гиббсовское состояние равновесия для данной системы частиц (в частности, что потенциал устойчив). Тогда при некоторых условиях, например при малой плотности частиц, можно доказать, что в любой момент времени с вероятностью единица все кластеры конечны. Более того, некоторые расширенные кластеры (названные в [23] динамическими кластерами) остаются конечными на определенном промежутке времени (см. [23]).

Однако другие проблемы, например о временах существования кластеров, пока открыты. Так, (устойчивые) связанные состояния, по-видимому, отсутствуют с вероятностью 1, помимо, конечно, одночастичных кластеров. Действительно, пусть имеется только два типа частиц 1,2 и потенциал $V_{12}$ таков, что единственно возможное связанное состояние состоит из двух частиц, где одна из них вращается вокруг другой по круговой орбите. При этом должно выполняться соотношение между скоростью этого вращения и расстоянием (радиусом круговой орбиты) между частицами. Но, так как распределения координат и скоростей независимы, это возможно только с нулевой вероятностью.

С другой стороны, существует бесконечное число метастабильных кластеров, где эти соотношения выполняются приближенно, и кластер живет достаточно долго, исчезая либо сам собой, либо из-за столкновений с другими кластерами. Интересной задачей является оценка времен жизни кластеров в зависимости от параметров гиббсовского распределения.

Есть и множество других открытых вопросов о связи понятий частицы в конечных и бесконечных системах.

В конечных системах частиц, как классических, так и квантовых, сначала вводятся элементарные частицы. В классическом случае - как точечные частицы, в квантовом - как операторы рождения-уничтожения. Гамильтониан же может иметь связанные состояния. В классическом случае это орбиты определенного вида, в квантовом - собственные функции дискретного спектра. В ряде случаев можно доказать, что они полностью определяют асимптотические по времени состояния системы. В принципе, могут быть связанные состояния любого числа частиц.

Если же мы рассматриваем бесконечную систему частиц, то определенная теория имеется только для равновесной динамики, т. е. динамики системы в равновесном (гиббсовском) состоянии. Здесь также вводятся элементарные, называемые голыми или неперенормированными, частицы, которые, однако, в отличие от конечных систем, уже не принадлежат дискретному спектру динамики после термодинамического предельного перехода. Появляется новый дискретный спектр (точнее, одночастичные состояния). Основная гипотеза, доказанная в некоторых случаях, состоит в том, что пара (гильбертово пространство, гамильтониан), соответствующая данной бесконечной системе взаимодействующих частиц, унитарно эквивалентна аналогичной паре для неко- 
торой системы невзаимодействующих частиц, называемых тогда квазичастицами. Эти квазичастицы могут быть близки к первоначально введенным голым частицам, например если взаимодействие мало. В физике квазичастицы часто представляются как голая частица, собирающая вокруг себя облако из других голых частиц. Это могло бы объяснить связь между квазичастицами в бесконечных системах и метастабильными состояниями конечных систем, но точных доказательств и даже точных формулировок здесь нет.

\section{Список литературы}

[1] A. Arkin, J. Ross, H. H. McAdams, "Stochastic kinetic analysis of developmental pathway bifurcation in phage $\lambda$-infected Escherichia coli cells", Genetics, 149:4 (1998), 1633-1648.

[2] H. H. McAdams, A. Arkin, "Stochastic mechanisms in gene expression", Proc. Natl. Acad. Sci. USA, 94:3 (1997), 814-819.

[3] Ch. Gadgil, Chang-Hyeong Lee, H. G. Othmer, "A stochastic analysis of first-order reaction networks", Bull. Math. Biol., 67:5 (2005), 901-946.

[4] И. В. Березин, А. А. Клесов, Практический курс химический и ферментативной кинетики, МГУ, М., 1978.

[5] V. A. Malyshev, S. A. Pirogov, A. N. Rybko, "Random walks and chemical networks", Mosc. Math. J., 4:2 (2004), 441-453.

[6] М. А. Леонтович, “Основные уравнения кинетической теории газов с точки зрения теории случайных процессов”, ЖЭТФ, 5:3-4 (1935), 211-231.

[7] D. A. McQuarrie, "Stochastic approach to chemical kinetics", J. Appl. Probability, 4:3 (1967), 413-478.

[8] А.В. Калинкин, УМH, 57:2 (2002), 23-84; англ. пер.: A. V. Kalinkin, "Markov branching processes with interaction", Russian Math. Surveys, 57:2 (2002), 241-304.

[9] P. Whittle, Systems in stochastic equilibrium, Wiley Ser. Probab. Math. Stat.: Appl. Probab. Stat., Wiley, Chichester, 1986.

[10] R. D. Nelson, "The mathematics of product form queueing networks", ACM Comput. Service, 25:3 (1993), 339-369.

[11] F.P. Kelly, Reversibility and stochastic networks, Wiley Ser. Probab. Math. Stat.: Appl. Probab. Stat., Wiley, Chichester, 1979.

[12] Е. М. Лифшиц, Л. П. Питаевский, Теоретическая физика, т. 10: Физическая кинетика, Наука, М., 1979.

[13] V. A. Malyshev, "Microscopic models for chemical thermodynamics", J. Stat. Phys., 119:5-6 (2005), 997-1026.

[14] В. В. Веденяпин, Кинетические уравнения Больцмана и Власова, Физматлит, М., 2001.

[15] Я. Г. Батищева, В.В.Веденяпин, "Второй закон термодинамики для химической кинетики", Матем. моделирование, 17:8 (2005), 106-110.

[16] М. В.Волькенштейн, Физика и биология, Наука, М., 1980; англ. пер.: M. V. Volkenshtein, Physics and biology, Academic Press, New York, 1982.

[17] Дж. Марри, Нелинейнье дифференииальные уравнения в биологии, Мир, М., 1983; англ. пер. J. D. Murray, Lectures on nonlinear differential equation models in biology, Clarendon Press. XIII, Oxford, England, 1977.

[18] S. N. Ethier, Th. G. Kurtz, Markov processes. Characterization and convergence, Wiley Ser. Probab. Math. Stat.: Appl. Probab. Stat., Wiley, New York, 1986.

[19] K. Ball, Th. G. Kurtz, L. Popovic, G. Rempala, "Asymptotic analysis of multiscale approximations to reaction networks", Ann. Appl. Probab., 16:4 (2006), 1925-1961; arXiv: math/0508015. 
[20] H. Spohn, Fluctuation theory for the Boltzmann equation, Stud. Statist. Mech., 10, North-Holland, Amsterdam, 1983.

[21] V. A. Malyshev, "Fixed points for stochastic open chemical systems", Markov Process. Related Fields, 11:2 (2005), 337-354.

[22] G. Fayolle, V. Malyshev, S. Pirogov, "Stochastic chemical kinetics with energy parameters", Mathematics and computer science. III, Trends Math., Birkhäuser, Basel, 2004, 517-529.

[23] V.A. Malyshev, "Dynamical clusters of infinite particle dynamics", J. Math. Phys., 46:7 (2005), 073302.

[24] O. E. Lanford, "Time evolution of large classical systems", Dynamical systems, theory and applications (Seattle, 1974), Lecture Notes in Phys., 38, Springer, Berlin, 1975, $1-111$.

[25] H. Spohn, "Large scale dynamics of interacting particles", Texts Monogr. Phys., Springer, Berlin, 1991.

[26] E. Caglioti, C. Marchioro, M. Pulvirenti, "Non-equilibrium dynamics of three-dimensional infinite particle systems", Comm. Math. Phys., 215:1 (2000), 25-43.

[27] S. Caprino, M. Pulvirenti, "A cluster expansion approach to a one-dimensional Boltzmann equation: a validity result", Comm. Math. Phys., 166:3 (1995), 603-631.

[28] S. Caprino, M. Pulvirenti, "The Boltzmann-Grad limit for a one-dimensional Boltzmann equation in a stationary state", Comm. Math. Phys., 177:1 (1996), 63-81.

[29] S. Caprino, M. Pulvirenti, W. Wagner, "Stationary particle systems approximating stationary solutions to the Boltzman equation", SIAM J. Math. Anal., 29:4 (1998), 913-934.

[30] C. Cercignani, R. Illner, M. Pulvirenti, The mathematical theory of dilute gases, Appl. Math. Sci., 106, Springer, New York, 1994.

[31] C. Cercignani, V.I. Gerasimenko, D. Ya. Petrina, Many-particle dynamics and kinetic equations, Math. Appl., 420, Kluwer, Dordrecht, 1997.

[32] A. DeMasi, R. Esposito, J. L. Lebowitz, E. Presutti, "Hydrodynamics of stochastic cellular automata", Comm. Math. Phys., 125:1 (1989), 127-145.

[33] L. Arnold, M. Theodosopulu, "Deterministic limit of the stochastic model of chemical reactions with diffusion", Adv. Appl. Probab., 12:2 (1980), 367-379.

[34] M. Bramson, J. Lebowitz, "Spatial structure in low dimensions for diffusion limited two-particle reactions", Ann. Appl. Probab., 11:1 (2001), 121-181.

[35] К. Хепп, "Результаты и проблемы в необратимой статистической механике открытых систем", Гиббсовские состояния в статистической физике, Математика. Новое в зарубежной науке, 11, Мир, М., 1978; пер. с англ.: К. Нерp, "Results and problems in irreversible statistical mechanics of open systems", International Symposium on Mathematical Problems in Theoretical Physics (Kyoto Univ., Kyoto, 1975), Lecture Notes in Phys., 39, Springer, Berlin, 1975, 138-150.

[36] М. Рид, Б. Саймон, Методы современной математической физики. Теория рассеяния, III, Мир, М., 1982; пер. с англ.: M. Reed, B. Simon, Methods of modern mathematical physics. Scattering theory, III, Academic Press, New York-London, 1979.

[37] В. М. Алексеев, Лекиии по небесной механике, Регулярная и хаотическая динамика, НИТЦ, М., 2001.

В. А. Малышев (V. A. Malyshev)

Поступила в редакцию

Московский государственный университет

04.09.2007

им. М. В. Ломоносова

E-mail: malyshev2@yahoo.com

\section{С. А. Пирогов (S. А. Pirogov)}

Институт проблем передачи информации РАН

E-mail: pirogov@mail.ru 